\title{
Time-dependent model of mixed electroosmotic/pressure- driven three immiscible fluids in a rectangular microchannel
}

\author{
Li Haiwang, Teck Neng Wong", Nam-Trung Nguyen \\ School of Mechanical and Aerospace Engineering, Nanyang Technological University, 50 \\ Nanyang Avenue, Singapore 639798, Singapore \\ *Correponding author. E-mail address: mtnwong@ntu.edu.sg (T.N. Wong)
}

\begin{abstract}
:
The time-dependent aspects of pressure-driven three-liquid flow under the effect of electroosmotic flow (EOF) are analytically studied, in which non-conducting liquid is delivered by the pressure gradient and the interfacial viscous forces of two conducting liquids, the two conducting liquids are driven by electroosmosis and pressure gradient. The flow of the three liquids depends on the coupling effects between them, which involve the electrokinetic effect. The surface charges at the liquid-liquid interface are accounted in this model. At the interface, the shear stress is not continuous because of the presence of the surface charges. With Laplace transform method, an analytical solution of such time-dependent three-liquid flow in rectangular channel is presented.
\end{abstract}

Keywords: Time-dependent; Three-fluid stratified flow; Electrical double layer; Electroosmotic focusing.

\section{Introduction}

The microfluidic field, such as micro-TAS (total analysis systems), lab-on-a-chip and microreactors, has been widely developed during this decade. When two or more liquids flow in parallel into a microchannel, laminar fluid interfaces are generated due to low Reynolds number. The interface and the width of the focused stream are important for separation, reaction and mixing of chemical. The interface control is useful for focusing sample fluid or flow switching. The flow focusing technique provides a particularly effective means of controlling the passage of chemical reagents or bio-samples in a microchannel network, and has been successfully demonstrated in a wide variety of applications such as cell handing and analysis [1], biomedical and biopower systems [2], clinical diagnosis [3], immunoassays [4], DNA [5], proteins [6], other bioassays [7], environmental concerns and gas analysis $[8,9]$.

Using "flow-rate-ratio" method, hydrodynamic force is used for flow focusing [10]. Wu and Nguyen [11] theoretically studied the hydrodynamic focusing inside a microchannel. 
This paper studies the time-depend aspects of three-fluid electroosmotic flow considering the free charges at the interface by the analytical method. Because of the "excess" surface charge at the interfaces, the interfaces between the three flow are described as two dividing surface. Under the Debye-Hückel linear approximate, the analytical solution of the Poisson-Boltzmann equation is presented in this paper. Using Laplace transform, the exact solutions of the velocities and the flow rates are obtained for Navier-Stokes equation governing this three-liquid flow.

\section{Theoretical models}

To analyze the system, a Cartesian coordinate system $(x, y, z)$ is used where the origin point, $\mathrm{O}$, is set to be at the centre of the non-conducting fluid and the symmetric line is shown in Fig. 1. Planar interfaces are assumed. The heights of the conducting fluids and of the non-conducting fluid are denoted as $h_{1}, h_{3}$ and $2 h$, respectively. Half of the width of the channel is denoted by $w$. The aspect ratio is defined as $\chi=\left(h_{1}+h_{3}+2 h\right) / 2 w$. As a result of surface charge, electric double layers (EDLs) form near the liquid-liquid interface and the channel wall that is in contact with the conducting liquid. For a more general situation, the walls of the microchannel may be made of different materials, so that the zeta potentials at the bottom and top walls are $\xi_{1}$ and $\xi_{4}$, respectively, at the side walls as $\xi_{2}$ and $\xi_{5}$, respectively. The zeta potentials at the interfaces are $\xi_{3}$ and $\xi_{6}$. The electroosmotic flows are along the $x$ direction. Due to symmetry, only half of the cross section $(z>0)$ of the rectangular channel is considered.

\subsection{Electric double layers in the conducting liquid and surface electric charges at the interface}

When the electrolyte solutions contact with the wall or the immiscible fluids, the electric double layer is developed spontaneously near the wall or at the liquid-liquid interface [25]. Due to the presence of the EDL, the electric potential distribution, $\psi$, is determined by the wellknown Poisson-Boltzmann equation,

$\nabla^{2} \psi=\left(2 z_{0} e n_{0}\right) / \varepsilon \sinh \left(z_{0} e \psi / k_{b} T\right)$

where $z_{0}$ is the valence of ion, $e$ is the elementary charge, $n_{0}$ is the ionic number concentration in the bulk, $\varepsilon$ is the permittivity of the solution, $k_{b}$ is Boltzmann constant, and $T$ is absolute temperature.

The local volumetric net charge density, $\rho_{\mathrm{q}}$, is given by the Boltzmann distribution based on the assumption of local thermodynamic equilibrium,

$\nabla^{2} \psi=-\rho_{q} / \varepsilon$

Integrating Eqs. (1) and (2), we can obtain that

$\rho_{q}=-2 z_{0} e n_{0} \sinh \left(z_{0} e \psi / k_{b} T\right)$ 
In Eq. (3), because the EDL thickness and the zeta potential are small, it is assumed that the distribution of the electric charge density is not affected by the external electric field [26]. In addition, the effect of liquid motion on the charge redistribution is negligible because the liquid velocity in microchannels is very small.

The dimensionless form of Eq. (1) is

$$
\nabla^{2} \bar{\psi}=K^{2} \sinh (\bar{\psi})
$$

where $\bar{\psi}=z_{0} e \psi / k_{b} T, K=L_{\text {ref }} \kappa$ is the ratio of the length scale $L_{\text {ref }}$ to the characteristic double-layer thickness $1 / \kappa$. For this case, the reference length is chosen as $L_{\text {ref }}=w$. Here, $\kappa$ is the Debye-Hückel parameter,

$$
1 / \kappa=\left(\varepsilon k_{b} T / 2 z_{0}^{2} e^{2} n_{0}\right)^{1 / 2}
$$

For a small zeta potential $(0<\bar{\psi}<1)$, the electric potentials due to the charged wall are described by the linear Poisson-Boltzmann equation which can be written in terms of dimensionless variables as

$$
\nabla^{2} \bar{\psi}=K^{2} \bar{\psi}
$$

Based on the linear approximation, the dimensionless volumetric charge density is given by

$$
\bar{\rho}_{q}=-\bar{\psi}(\bar{z}, \bar{y})
$$

Due to the symmetry of the EDL fields in the rectangular channel, Eq. (6) is subjected to the following boundary conditions:

(for conducting liquid 1) $\begin{cases}\partial \bar{\psi}_{1} / \partial \bar{z}=0 & \text { at } \bar{z}=0 \\ \bar{\psi}_{1}=\bar{\xi}_{2} & \text { at } \bar{z}=\bar{w} \\ \bar{\psi}_{1}=\bar{\xi}_{3} & \text { at } \bar{y}=-\bar{h} \\ \bar{\psi}_{1}=\bar{\xi}_{1} & \text { at } \bar{y}=-\bar{h}_{1}-\bar{h}\end{cases}$

(for conducting liquid 3) $\begin{cases}\partial \bar{\psi}_{3} / \partial \bar{z}=0 & \text { at } \bar{z}=0 \\ \bar{\psi}_{3}=\bar{\xi}_{5} & \text { at } \bar{z}=\bar{w} \\ \bar{\psi}_{3}=\bar{\xi}_{6} & \text { at } \bar{y}=\bar{h} \\ \bar{\psi}_{3}=\bar{\xi}_{4} & \text { at } \bar{y}=\bar{h}_{3}+\bar{h}\end{cases}$

The solutions to the Poisson-Boltzmann equation subjected to the above boundary conditions are obtained as

$$
\begin{aligned}
\bar{\psi}_{1}= & \sum_{j=0}^{\infty} \frac{4 \bar{\xi}_{1}}{(2 j+1) \pi}(-1)^{j+1} \frac{\sinh \left[B_{j}(\bar{h}+\bar{y})\right]}{\sinh \left(B_{j} \bar{h}_{1}\right)} \cos \left(\lambda_{j} \bar{z}\right)-\sum_{j=0}^{\infty} \\
& \times \frac{4 \bar{\xi}_{3}}{(2 j+1) \pi}(-1)^{j+1} \times \frac{\sinh \left[B_{j}\left(\bar{h}_{1}+\bar{h}+\bar{y}\right)\right]}{\sinh \left[B_{j} \bar{h}_{1}\right]} \cos \left(\lambda_{j} \bar{z}\right) \\
& +\sum_{p=1}^{\infty} \frac{2 \bar{\xi}_{2}}{p \pi}\left[(-1)^{p}-1\right] \frac{\cosh \left(A_{p 1} \bar{z}\right)}{\cosh \left(A_{p 1} \bar{w}\right)} \sin \left[\frac{p \pi}{\bar{h}_{1}}(\bar{y}+\bar{h})\right]
\end{aligned}
$$


for conducting liquid 1 , and

$$
\begin{aligned}
\bar{\psi}_{3} & =-\sum_{j=0}^{\infty} \frac{4 \bar{\xi}_{4}}{(2 j+1) \pi} \times(-1)^{j+1} \times \frac{\sinh \left[B_{j}(\bar{y}-\bar{h})\right]}{\sinh \left(B_{j} \bar{h}_{3}\right)} \cos \left(\lambda_{j} \bar{z}\right) \\
& +\sum_{j=0}^{\infty} \frac{4 \bar{\xi}_{6}}{(2 j+1) \pi} \times(-1)^{j+1} \times \frac{\sinh \left[B_{j}\left(\bar{y}-\bar{h}_{3}-\bar{h}\right)\right]}{\sinh \left[B_{j} \bar{h}_{3}\right]} \cos \left(\lambda_{j} \bar{z}\right) \\
& -\sum_{p=1}^{\infty} \frac{2 \bar{\xi}_{5}}{p \pi}\left[(-1)^{p}-1\right] \frac{\cosh \left(A_{p 3} \bar{z}\right)}{\cosh \left(A_{p 3} \bar{w}\right)} \sin \left[\frac{p \pi}{\bar{h}_{3}}(\bar{y}-\bar{h})\right]
\end{aligned}
$$

for conducting liquid 3 , where $\lambda_{j}=(2 j-1) \pi / 2 \bar{w} \quad, \quad B_{j}=\sqrt{K^{2}+\lambda_{j}^{2}}$, $A_{p 1}=\sqrt{K^{2}+\left(p \pi / \bar{h}_{1}\right)^{2}}, A_{p 3}=\sqrt{K^{2}+\left(p \pi / \bar{h}_{3}\right)^{2}}$.

In the above discussion of electroosmosis, the charge state of the surface is described in terms of surface potential at the shear plane, which is identified with the zeta potential [27]. This surface potential is related to a certain charge density at the surface [28]. From electrostatics, the normal component of the gradient of the electric potential, $\psi$, jumps by an amount proportional to the surface charge density, $\rho_{q}^{S}$. That is

$\rho_{q}^{s}=-\varepsilon \partial \psi / \partial y$

It is assumed that the gradient of electric potential in the nonconducting liquid vanishes. Using the reference surface charge density as $\left(\varepsilon k_{b} T\right) /\left(L_{\mathrm{ref}} Z_{0} e\right)$ we obtain the dimensionless surface charge densities at the two-liquid interface as

$$
\begin{aligned}
\bar{\rho}_{q 1}^{s}(\bar{z})= & -\sum_{j=1}^{\infty} \frac{4(-1)^{j+1} B_{j}}{(2 j+1) \pi}\left[\frac{\bar{\xi}_{1}}{\sinh \left(B_{j} \bar{h}_{1}\right)}-\frac{\bar{\xi}_{3}}{\tanh \left(B_{j} \bar{h}_{1}\right)}\right] \cos \left(\lambda_{j} \bar{z}\right) \\
& -\sum_{p=1}^{\infty} \frac{2 \bar{\xi}_{2}}{\bar{h}_{1}}\left[(-1)^{p}-1\right] \frac{\cosh \left(A_{p 1} \bar{z}\right)}{\cosh \left(A_{p 1} \bar{w}\right)}
\end{aligned}
$$

for the surface charge at interface $1-2$, and

$$
\begin{aligned}
\bar{\rho}_{q 3}^{s}(\bar{z})= & \sum_{j=1}^{\infty} \frac{4(-1)^{j+1} B_{j}}{(2 j+1) \pi}\left[\frac{\bar{\xi}_{4}}{\sinh \left(B_{j} \bar{h}_{3}\right)}-\frac{\bar{\xi}_{6}}{\tanh \left(B_{j} \bar{h}_{3}\right)}\right] \cos \left(\lambda_{j} \bar{z}\right) \\
& +\sum_{p=1}^{\infty} \frac{2 \bar{\xi}_{5}}{\bar{h}_{3}}\left[(-1)^{p}-1\right] \frac{\cosh \left(A_{p 3} \bar{z}\right)}{\cosh \left(A_{p 3} \bar{w}\right)}
\end{aligned}
$$

for the surface charge at interface $2-3$.

The solutions of Eqs. (13) and (14) show that the contributions of zeta potential at the top/bottom walls, $\xi_{4}$ and $\xi_{1}$, are relatively small and the contributions of the side walls, $\xi_{2}$ and $\xi_{5}$ are also relatively small except when $z$ approaches to $w$. The volumetric net charge density, Eq. (7), and the interface charge density, Eqs. (13) and(14), are required to determine the electrostatic force caused by the presence of zeta potential. The bulk electrostatic force is considered as an additional body force exerting on the conducting liquid in the conventional 
Navier-Stokes equation. Therefore, the conducting liquids are under the action of pressure gradient, electrostatic force and the viscous shear force at the interface. Similarly, the non-conducting liquid flows as a result of pressure gradient and external electrostatic force due to the electrokinetic charge density at the interface, which will be discussed in the following section.

\subsection{Momentum equation of the three-liquid flow}

We now consider the transient pressure-driven three-liquid, under the influence of two uniform external electric fields, $E_{x 1}$ and $E_{x 3}$, through a rectangular channel as illustrated in Fig. 2. The Navier-Stokes equation for an incompressible laminar liquid is given by

$\bar{\rho} \frac{\partial \overline{\mathbf{V}}}{\partial t}+\bar{\rho}(\overline{\mathbf{V}} \cdot \nabla) \overline{\mathbf{V}}=-\nabla \bar{p}+\overline{\mathbf{F}}+\frac{1}{\operatorname{Re}} \bar{\mu} \nabla^{2} \overline{\mathbf{V}}$

where, $\mathbf{V}$ is the velocity vector, $p$ is the pressure, $\rho$ is the density, and $\mu$ is dynamic viscosity of the fluid. Ignoring the gravity effect, the force $\mathbf{F}$ is caused by the action of the induced electrical field, $E_{x}$, and the net charge density, $\rho_{e}(y, z)$. This force exerts only in the electric double-layer regions of the conducting fluid flow along the $x$-direction. For the conducting fluid,$\overline{\mathbf{F}}=G_{x} \bar{\rho}_{\mathrm{q}}$, and for the the non-conducting fluid, $\mathbf{F}=0$.

To evaluate the electrokinetic effects, it is assumed that the flow is formed by three simple immiscible Newtonian liquids with constant viscosities, which are independent of shear rate and the local electric field strength. The flow is a transient, fully developed, laminar stratified flow. The pressure gradient is assumed to be constant along the channel and the pressure gradient along $y$ and $z$ directions are both zero.

As EDLs form in the conducting liquid flow, for fully developed flow, the momentum equations of the three liquids reduce to

(conducting fluid 1) $\frac{\partial \bar{u}_{1}}{\partial \bar{t}}=\frac{1}{\operatorname{Re}}\left(\frac{\partial^{2} \bar{u}_{1}}{\partial \bar{y}^{2}}+\frac{\partial^{2} \bar{u}_{1}}{\partial \bar{z}^{2}}\right)+G_{x 1} \bar{\rho}_{q 1}-\frac{d \bar{p}}{d \bar{x}}$

(non-conducting fluid 2) $\bar{\rho}_{2} \frac{\partial \bar{u}_{2}}{\partial \bar{t}}=\frac{\bar{\mu}_{2}}{\operatorname{Re}}\left(\frac{\partial^{2} \bar{u}_{2}}{\partial \bar{y}^{2}}+\frac{\partial^{2} \bar{u}_{2}}{\partial \bar{z}^{2}}\right)-\frac{d \bar{p}}{d \bar{x}}$

(conducting fluid 3) $\bar{\rho}_{3} \frac{\partial \bar{u}_{3}}{\partial \bar{t}}=\frac{\bar{\mu}_{3}}{\operatorname{Re}}\left(\frac{\partial^{2} \bar{u}_{3}}{\partial \bar{y}^{2}}+\frac{\partial^{2} \bar{u}_{3}}{\partial \bar{z}^{2}}\right)+G_{x 3} \bar{\rho}_{q 3}-\frac{d \bar{p}}{d \bar{x}}$

where $G_{x 1}=\left(2 z_{0} e n_{0} L_{\mathrm{ref}} E_{x 1}\right) /\left(\rho_{\mathrm{ref}} U_{\mathrm{ref}}^{2}\right), G_{x 3}=\left(2 z_{0} e n_{0} L_{\mathrm{ref}} E_{x 3}\right) /\left(\rho_{\mathrm{ref}} U_{\mathrm{ref}}^{2}\right)$, the subscripts, 1,2 and 3 , denote the conducting liquid 1 , non-conducting fluid 2 and the conducting liquid 3 , respectively. $u$ is the velocity along $x$-direction. $\rho_{\mathrm{e} 1}$ and $\rho_{\mathrm{e} 3}$ are the local volumetric net charge densities shown by Eq. (3), respectively. The reference $L_{\text {ref }}$ is the hydraulic diameter of the rectangular channel. The reference velocity, $U_{\text {ref }}=\left(E_{x 1} \varepsilon_{0} \varepsilon_{r} \xi_{\text {ref }}\right) / \mu_{\text {ref }}$; the reference time, $\bar{t}=L_{r e f}^{2}$ / $v_{1}$; the reference viscosity, $\mu_{\text {ref }}=\mu_{1}$, the reference density, $\rho_{\text {ref }}=\rho_{1}$; the kinematics viscosity 
ratio $\alpha_{2}=v_{2} / v_{1}, \alpha_{3}=v_{3} / v_{1}$, the dynamic viscosity ratio, $\bar{\mu}_{1}=\beta_{1}=1, \bar{\mu}_{2}=\beta_{2}=\mu_{2} / \mu_{1}$ and $\bar{\mu}_{3}=\beta_{3}=\mu_{3} / \mu_{1}$; the Reynolds number is defined as $R e=\left(\rho_{\text {ref }} U_{\text {ref }} L_{\text {ref }}\right) / \mu_{\text {ref }}$.

At the interface, matching conditions must be obeyed. There are the continuities of velocity $\bar{u}_{1}=\bar{u}_{2}$ (interface $1-2$ )

$\bar{u}_{2}=\bar{u}_{3}$ (interface $2-3$ )

and the shear stress balance, which jumps abruptly at the interface because of the presence of the certain surface charge density,

$\bar{\mu}_{1} \frac{\partial \bar{u}_{1}}{\partial \bar{y}}+M_{1} \bar{\rho}_{q 1}^{s}=\bar{\mu}_{2} \frac{\partial \bar{u}_{2}}{\partial \bar{y}}, \quad$ at $\bar{y}=-\bar{h}$ (interface $\left.1-2\right)$

$$
\bar{\mu}_{3} \frac{\partial \bar{u}_{3}}{\partial \bar{y}}+M_{3} \bar{\rho}_{q 3}^{s}=\bar{\mu}_{2} \frac{\partial \bar{u}_{2}}{\partial \bar{y}}, \quad \text { at } \bar{y}=\bar{h} \text { (interface } 2-3 \text { ) }
$$

where $y$ is the direction normal to the interface of the two liquids. The term $M_{1} \bar{\rho}_{q 1}^{\mathrm{s}}$ and $M_{3} \bar{\rho}_{q 3}^{\mathrm{s}}$ account for the force on the interface 1-2 and interface 2-3 due to the electrokinetic interface charges, respectively.

The dimensionless matching conditions become

(interface 1-2) $\left\{\begin{array}{l}\bar{u}_{1}(\bar{z},-\bar{h}, \bar{t})=\bar{u}_{2}(\bar{z},-\bar{h}, \bar{t}) \\ \frac{\partial \bar{u}_{1}(\bar{z},-\bar{h}, \bar{t})}{\partial \bar{y}}=\beta_{2} \frac{\partial \bar{u}_{2}(\bar{z},-\bar{h}, \bar{t})}{\partial \bar{y}}-M_{1} \bar{\rho}_{q 1}^{s}(\bar{z})\end{array}\right.$

(interface 2-3) $\left\{\begin{array}{l}\bar{u}_{3}(\bar{z}, \bar{h}, \bar{t})=\bar{u}_{2}(\bar{z}, \bar{h}, \bar{t}) \\ \frac{\partial \bar{u}_{3}(\bar{z}, \bar{h}, \bar{t})}{\partial \bar{y}}=\left(\beta_{2} / \beta_{3}\right) \frac{\partial \bar{u}_{2}(\bar{z}, \bar{h}, \bar{t})}{\partial \bar{y}}-M_{3} \bar{\rho}_{q 3}^{s}(\bar{z})\end{array}\right.$

where $M_{1}=\left(\varepsilon k_{b} T E_{x 1}\right) /\left(z_{0} e U_{\text {ref }} \mu_{\text {ref }}\right)$ and $M_{3}=\left(\varepsilon k_{b} T E_{x 3}\right) /\left(z_{0} e U_{\text {ref }} \mu_{\text {ref }}\right), M_{1}$ and $M_{2}$ are the electrokinetic effects in the matching conditions.

In the rectangular-cross-section channel, the dimensionless boundary conditions for fluids 1,2 and 3 are:

(conducting fluid 1) $\begin{cases}\frac{\partial \bar{u}_{1}}{\partial \bar{z}}=0 & \text { when } \bar{z}=0 \\ \bar{u}_{1}=0 & \text { when } \bar{z}=\bar{w} \\ \bar{u}_{1}=0 & \text { when } \bar{y}=-\bar{h}-\bar{h}_{1} \\ \bar{u}_{1}=0 & \text { when } \bar{t}=0\end{cases}$

The dimensionless boundary conditions for the non-conducting liquid 2 are

(non-conducting fluid 2) $\begin{cases}\frac{\partial \bar{u}_{2}}{\partial \bar{z}}=0 & \text { when } \bar{z}=0 \\ \bar{u}_{2}=0 & \text { when } \bar{z}=\bar{w} \\ \bar{u}_{2}=0 & \text { when } \bar{t}=0\end{cases}$ 
The dimensionless boundary conditions for the non-conducting liquid 3 are

(conducting fluid 3) $\begin{cases}\frac{\partial \bar{u}_{3}}{\partial \bar{z}}=0 & \text { when } \bar{z}=0 \\ \bar{u}_{3}=0 & \text { when } \bar{z}=\bar{w} \\ \bar{u}_{3}=0 & \text { when } \bar{y}=\bar{h}_{3}+\bar{h} \\ \bar{u}_{3}=0 & \text { when } \bar{t}=0\end{cases}$

Due to linearity, the velocities of the conducting liquids and the non-conducting liquid (Eqs. (16)-(18)) can be decomposed into two parts:

$\bar{u}=\bar{u}^{p}+\bar{u}^{E}$

where $\bar{u}^{E}$ corresponds to the velocity driven by electroosmotic force, and $\bar{u}^{p}$ is the velocity driven by pressure gradient.

The dimensionless momentum equations can be presented as

$$
\begin{gathered}
\left\{\begin{array}{l}
\operatorname{Re} \frac{\partial \bar{u}_{1}^{E}}{\partial t}=\frac{\partial^{2} \bar{u}_{1}^{E}}{\partial \bar{z}^{2}}+\frac{\partial^{2} \bar{u}_{1}^{E}}{\partial \bar{y}^{2}}+\operatorname{Re} G_{x 1} \bar{\rho}_{q 1} \\
\operatorname{Re} \frac{\partial \bar{u}_{1}^{P}}{\partial t}=\frac{\partial^{2} \bar{u}_{1}^{P}}{\partial \bar{z}^{2}}+\frac{\partial^{2} \bar{u}_{1}^{p}}{\partial \bar{y}^{2}}-\operatorname{Re} \frac{d \bar{P}}{d \bar{x}}
\end{array}\right\} \text { (conducting liquid 1) } \\
\left\{\begin{array}{l}
\frac{\operatorname{Re}}{\alpha_{2}} \frac{\partial \bar{u}_{2}^{E}}{\partial t}=\frac{\partial^{2} \bar{u}_{2}^{E}}{\partial \bar{z}^{2}}+\frac{\partial^{2} \bar{u}_{2}^{E}}{\partial \bar{y}^{2}} \\
\frac{\operatorname{Re}}{\alpha_{2}} \frac{\partial \bar{u}_{2}^{P}}{\partial t}=\frac{\partial^{2} \bar{u}_{2}^{P}}{\partial \bar{z}^{2}}+\frac{\partial^{2} \bar{u}_{2}^{P}}{\partial \bar{y}^{2}}-\frac{\operatorname{Re}}{\beta_{2}} \frac{d \bar{P}}{d \bar{x}}
\end{array}\right\} \text { (non-conducting liquid 2) }
\end{gathered}
$$

and

$$
\left\{\begin{array}{l}
\frac{\operatorname{Re}}{\alpha_{3}} \frac{\partial \bar{u}_{3}^{E}}{\partial t}=\frac{\partial^{2} \bar{u}_{3}^{E}}{\partial \bar{z}^{2}}+\frac{\partial^{2} \bar{u}_{3}^{E}}{\partial \bar{y}^{2}}+\frac{\operatorname{Re}}{\beta_{3}} G_{x 3} \bar{\rho}_{q 3} \\
\frac{\operatorname{Re}}{\alpha_{3}} \frac{\partial \bar{u}_{3}^{P}}{\partial t}=\frac{\partial^{2} \bar{u}_{3}^{P}}{\partial \bar{z}^{2}}+\frac{\partial^{2} \bar{u}_{3}^{P}}{\partial \bar{y}^{2}}-\frac{\operatorname{Re}}{\beta_{3}} \frac{d \bar{P}}{d \bar{x}}
\end{array}\right\} \text { (conducting liquid 3) }
$$

The Laplace transform method is applied to solve these equations. This paper assumes that the zeta potentials are not affected by the external electric field. We substitute the local volumetric net charge density, Eq. (13), into momentum equation Eqs. (29), then the Laplace transform of the conducting fluid 1 is

$$
s \operatorname{Re} \bar{U}_{1}^{E}(\bar{z}, \bar{y}, s)=\left[\frac{\partial^{2} \bar{U}_{1}^{E}(\bar{z}, \bar{y}, s)}{\partial \bar{z}^{2}}+\frac{\partial^{2} \bar{U}_{1}^{E}(\bar{z}, \bar{y}, s)}{\partial \bar{y}^{2}}\right]-\frac{\operatorname{Re} G_{x 1} \bar{\psi}_{1}(\bar{z}, \bar{y})}{s}
$$

with the boundary conditions of

$$
\begin{cases}\frac{\partial \bar{U}_{1}^{E}(\bar{z}, \bar{y}, s)}{\partial \bar{z}}=0 & \text { when } \bar{z}=0 \\ \bar{U}_{1}^{E}(\bar{z}, \bar{y}, s)=0 & \text { when } \bar{z}=\bar{w} \\ \bar{U}_{1}^{E}(\bar{z}, \bar{y}, s)=0 & \text { when } \bar{y}=-\bar{h}-\bar{h}_{1}\end{cases}
$$


where $s$ is the Laplace transform parameter. The Laplace transforms of Eqs. (29)-(31) are

$s \operatorname{Re} \bar{U}_{1}^{P}(\bar{y}, \bar{z}, s)=\frac{\partial^{2} \bar{U}_{1}^{\mathrm{P}}(\bar{y}, \bar{z}, s)}{\partial \bar{z}^{2}}+\frac{\partial^{2} \bar{U}_{1}^{P}(\bar{y}, \bar{z}, s)}{\partial \bar{y}^{2}}-\frac{\operatorname{Re}(d \bar{P} / d \bar{x})}{s}$

with the boundary conditions of

$$
\begin{cases}\frac{\partial \bar{U}_{1}^{p}(\bar{z}, \bar{y}, s)}{\partial \bar{z}}=0 & \text { when } \bar{z}=0 \\ \bar{U}_{1}^{p}(\bar{z}, \bar{y}, s)=0 & \text { when } \bar{z}=\bar{w} \\ \bar{U}_{1}^{p}(\bar{z}, \bar{y}, s)=0 & \text { when } \bar{y}=-\bar{h}-\bar{h}_{1}\end{cases}
$$

The Laplace transforms of Eqs. (30) and (31) are

Non-conducting fluid 2

$$
\frac{s \operatorname{Re}}{\alpha_{2}} \bar{U}_{2}^{E}(\bar{z}, \bar{y}, s)=\frac{\partial^{2} \bar{U}_{2}^{E}(\bar{z}, \bar{y}, s)}{\partial \bar{z}^{2}}+\frac{\partial^{2} \bar{U}_{2}^{E}(\bar{z}, \bar{y}, s)}{\partial \bar{y}^{2}}
$$

with the boundary conditions of

$$
\begin{cases}\frac{\partial \bar{U}_{2}^{E}(\bar{z}, \bar{y}, s)}{\partial \bar{z}}=0 & \text { when } \bar{z}=0 \\ \bar{U}_{2}^{E}(\bar{z}, \bar{y}, s)=0 & \text { when } \bar{z}=\bar{w}\end{cases}
$$

and

$\frac{\partial^{2} \bar{U}_{2}^{p}(\bar{z}, \bar{y}, s)}{\partial \bar{z}^{2}}+\frac{\partial^{2} \bar{U}_{2}^{p}(\bar{z}, \bar{y}, s)}{\partial \bar{y}^{2}}=\frac{s \operatorname{Re}}{\alpha_{2}} \bar{U}_{2}^{p}(\bar{z}, \bar{y}, s)+\frac{\operatorname{Re}(d \bar{P} / d \bar{x})}{s \beta_{2}}$

with the boundary conditions of

$$
\begin{cases}\frac{\partial \bar{U}_{2}^{p}(\bar{z}, \bar{y}, s)}{\partial \bar{z}}=0 & \text { when } \bar{z}=0 \\ \bar{U}_{2}^{p}(\bar{z}, \bar{y}, s)=0 & \text { when } \bar{z}=\bar{w}\end{cases}
$$

Conducting fluid 3

$$
\frac{s \operatorname{Re}}{\alpha_{3}} \bar{U}_{3}^{E}(\bar{z}, \bar{y}, s)=\frac{\partial^{2} \bar{U}_{3}^{E}(\bar{z}, \bar{y}, s)}{\partial \bar{z}^{2}}+\frac{\partial^{2} \bar{U}_{3}^{E}(\bar{z}, \bar{y}, s)}{\partial \bar{y}^{2}}-\frac{\operatorname{Re}}{s \beta_{3}} G_{x 3} \bar{\psi}_{3}(\bar{z}, \bar{y})
$$


with the boundary conditions of

$\begin{cases}\frac{\partial \bar{U}_{3}^{E}(\bar{z} \bar{y}, s)}{\partial \bar{z}}=0 & \text { when } \bar{z}=0 \\ \bar{U}_{3}^{E}(\bar{z}, \bar{y}, s)=0 & \text { when } \bar{z}=\bar{w} \\ \bar{U}_{3}^{E}(\bar{z}, \bar{y}, s)=0 & \text { when } \bar{y}=\bar{h}+\bar{h}_{3}\end{cases}$

and

$\frac{\partial^{2} \bar{U}_{3}^{p}(\bar{z}, \bar{y}, s)}{\partial \bar{z}^{2}}+\frac{\partial^{2} \bar{U}_{3}^{p}(\bar{z}, \bar{y}, s)}{\partial \bar{y}^{2}}=\frac{s \operatorname{Re}}{\alpha_{3}} \bar{U}_{3}^{p}(\bar{z}, \bar{y}, s)+\frac{\operatorname{Re}(d \bar{P} / d \bar{x})}{s \beta_{3}}$

with the boundary conditions of

$$
\begin{cases}\frac{\partial \bar{U}_{3}^{p}(\bar{z}, \bar{y}, s)}{\partial \bar{z}}=0 & \text { when } \bar{z}=0 \\ \bar{U}_{3}^{p}(\bar{z}, \bar{y}, s)=0 & \text { when } \bar{z}=\bar{w} \\ \bar{U}_{3}^{p}(\bar{z}, \bar{y}, s)=0 & \text { when } \bar{y}=\bar{h}+\bar{h}_{3}\end{cases}
$$

Using the separation of variables method, the solution of Eq. (32) with the boundary of Eq. (33) gives as

$$
\begin{aligned}
& \bar{U}_{1}^{E}(\bar{z}, \bar{y}, s)=\sqrt{\frac{2}{\bar{w}}} \sum_{j=0}^{\infty}\left\{\left\{\tanh \left[D_{1 j}\left(\bar{h}+\bar{h}_{1}\right)\right] \cosh \left(D_{1 j} \bar{y}\right)+\sinh \left(D_{1 j} \bar{y}\right)\right\} b_{1 j}^{E}\right. \\
& \left.-\frac{\phi_{1 j}\left(-\bar{h}_{1}-\bar{h}, s\right)}{\cosh \left[D_{1 j}\left(\bar{h}+\bar{h}_{1}\right)\right]} \cosh \left(D_{1 j} \bar{y}\right)+\phi_{1 j}(\bar{y}, s)\right\} \cos \left(\lambda_{j} \bar{z}\right)
\end{aligned}
$$

Using the same method, the solutions of Eqs. 34, 36, 38, 40, 42 give, respectively, as

$$
\begin{aligned}
& \bar{U}_{1}^{P}(\bar{y}, \bar{z}, s)=\sqrt{\frac{2}{\bar{w}}} \sum_{j=0}^{\infty}\left\{\frac{\operatorname{Re}}{s \lambda_{j} D_{1 j}^{2}} \frac{d \bar{P}}{d \bar{x}} \sqrt{\frac{2}{\bar{w}}}(-1)^{j}\left\{\frac{\cosh \left(D_{1 j} \bar{y}\right)}{\cosh \left[D_{1 j}\left(\bar{h}_{1}+\bar{h}\right)\right]}-1\right\}\right. \\
& \left.+\left\{\tanh \left[D_{1 j}\left(\bar{h}_{1}+\bar{h}\right)\right] \cosh \left(D_{1 j} \bar{y}\right)+\sinh \left(D_{1 j} \bar{y}\right)\right\} b_{1 j}^{P}\right\} \cos \left(\lambda_{j} \bar{z}\right) \\
& \bar{U}_{2}^{E}(\bar{z}, \bar{y}, s)=\sqrt{\frac{2}{\bar{w}}} \sum_{j=0}^{\infty}\left[a_{2 j}^{E}(s) \cosh \left(E_{2 j}^{E} \bar{y}\right)+a_{2 j}^{E}(s) \sinh \left(E_{2 j}^{E} \bar{y}\right)\right] \cos \left(\lambda_{j} \bar{z}\right) \\
& \bar{U}_{2}^{p}(\bar{z}, \bar{y}, s)=\sum_{j=1}^{\infty}\left\{a_{2 j}^{p}(s) \cosh \left(E_{2 j} \bar{y}\right)+b_{2 j}^{p}(s) \sinh \left(E_{2 j} \bar{y}\right)-\operatorname{Re} \times \frac{d \bar{P}}{d \bar{x}}\right. \\
& \left.\times \sqrt{\frac{2}{\bar{w}}} \times \frac{(-1)^{j}}{s \beta_{2} \lambda_{j} E_{2 j}^{2}}\right\} \cos \left(\lambda_{j} \bar{z}\right)
\end{aligned}
$$




$$
\begin{aligned}
& \bar{U}_{3}^{E}(\bar{z}, \bar{y}, s)=\sum_{j=0}^{\infty}\left\{\left\{-\tanh \left[E_{3 j}\left(\bar{h}+\bar{h}_{3}\right)\right] \cosh \left(E_{3 j} \bar{y}\right)+\sinh \left(E_{3 j} \bar{y}\right)\right\} b_{3 j}^{E}\right. \\
& \left.-\frac{\phi_{3 j}\left(\bar{h}+\bar{h}_{3}, s\right)}{\cosh \left[E_{3 j}\left(\bar{h}+\bar{h}_{3}\right)\right]} \cosh \left(E_{3 j} \bar{y}\right)+\phi_{3 j}(\bar{y}, s)\right\} \cos \left(\lambda_{j} \bar{z}\right) \\
& \bar{U}_{3}^{P}(\bar{z}, \bar{y}, s)=\sqrt{\frac{2}{\bar{w}}} \sum_{j=0}^{\infty}\left\{\frac{1}{E_{3 j}^{2}} \frac{\operatorname{Re}}{\lambda_{j} s \beta_{3}} \frac{d \bar{P}}{d \bar{x}} \sqrt{\frac{2}{\bar{w}}}(-1)^{j}\left\{\frac{\cosh \left(E_{3 j} \bar{y}\right)}{\cosh \left[E_{3 j}\left(\bar{h}_{3}+\bar{h}\right)\right]}-1\right\}\right. \\
& \left.+\left\{-\tanh \left[E_{3 j}\left(\bar{h}_{3}+\bar{h}\right)\right] \cosh \left(E_{3 j} \bar{y}\right)+\sinh \left(E_{3 j} \bar{y}\right)\right\} b_{3 j}^{P}\right\} \cos \left(\lambda_{j} \bar{z}\right)
\end{aligned}
$$

where $D_{1 j}=\sqrt{\lambda_{j}^{2}+s \mathrm{R} e}, E_{2 j}=\sqrt{\lambda_{j}^{2}+s \mathrm{R} e / \alpha_{2}}, E_{3 j}=\sqrt{\lambda_{j}^{2}+s \mathrm{R} e / \alpha_{3}}$.

Integrate the matching conditions of Eqs. (23) and (24), the coefficients $b_{1 j}^{E}, a_{2 j}^{E}, b_{2 j}^{E}, b_{3 j}^{E}, b_{1 j}^{p}$, $a_{2 j}^{p}, b_{2 j}^{p}$, and $b_{3 j}^{p}$ can be obtained as

$b_{1 j}^{E}=b_{2 j}^{E} / A+H$

$a_{2 j}^{E}=J b_{1 j}^{E}+K b_{3 j}^{E}+L$

$b_{2 j}^{E}=C b_{3 j}^{E}+D b_{1 j}^{E}+G$

$b_{3 j}^{E}=b_{2 j}^{E} / B+I$

$b_{1 j}^{p}=\left(b_{2 j}^{p}-T\right) / S$

$a_{2 j}^{p}=N b_{3 j}^{P}+Q b_{1 j}^{p}+M$

$b_{2 j}^{p}=(O-R T / S-P U / V) /(1-R / S-P / V)$

$b_{3 j}^{P}=\left(b_{2 j}^{P}-U\right) / V$

The detailed mathematical derivation of the coefficients $\mathrm{A}$ to $\mathrm{V}, \phi_{1 j}, \phi_{3 j}$ is presented in Appendix A.

According $\bar{Q}_{j}^{E}(s)=2 \int_{-\bar{h}-\bar{h}_{1}}^{-\bar{h}} \int_{0}^{\bar{w}} \bar{U}_{j}^{E}(\bar{y}, \bar{z}, s) d \bar{z} d \bar{y}$ and $\bar{Q}_{j}^{p}(s)=2 \int_{-\bar{h}-\bar{h}_{1}}^{-\bar{h}} \int_{0}^{\bar{w}} \bar{U}_{j}^{p}(\bar{y}, \bar{z}, s) d \bar{z} d \bar{y}$, the dimensionless volumetric flow rates areobtained (Appendix A). 
To obtain the final results, it is required to applied inversions of the Laplace transform on velocities $\bar{U}^{E}(\bar{y}, \bar{z}, s), \bar{U}^{p}(\bar{y}, \bar{z}, s)$ and flow rates $\bar{Q}^{E}(s), \bar{Q}^{p}(s)$. Due to the complex nature of those forms, the numerical inversion of Laplace transforms method-Gaver-Stehfest method with double precision is used in this paper [29].

\section{Results and discussion}

In this paper, governing equations for the EDL distributions in the two conducting liquids, velocity profiles, flow rates for the three-fluid flow, were derived for a rectangular microchannel. The two conducting fluids hold the bottom and upper parts, the liquid fractions for conducting fluid 1 and conducting fluid 3 are 0.3 , respectively, and the non-conducting fluid holds the middle part of the channel, the liquid fraction is 0.4 . Many methods for determining the zeta potentials at the wall and at the interface were proposed [25]. The zeta potentials at the channel walls, $\bar{\xi}_{\text {wall }}\left(\bar{\xi}_{1}, \bar{\xi}_{2}, \bar{\xi}_{4}, \bar{\xi}_{5}\right)$ depend on the material properties of the wall and the ionic properties of the fluid [12]. We choose the walls zeta potentials to be the same at $\bar{\xi}_{\text {wall }}\left(\bar{\xi}_{1}, \bar{\xi}_{2}, \bar{\xi}_{4}, \bar{\xi}_{5}\right)=-25 \mathrm{mV}$ as the default value. The zeta potential between two immiscible liquids, $\bar{\xi}_{3}$ and $\bar{\xi}_{6}$, does not only depend on the ionic properties of two fluids, but also on the $\mathrm{pH}$ and the concentration of the electrolyte [30,31]. The dimensionless parameter $K$ is defined as $K=\kappa D_{h}$ to evaluate parameters affecting the EDL profiles. $1 / \kappa$ refers to the characteristic thickness of the EDL. As the Debye-Hückel parameter in Eq. (5) is proportional to the square root of the bulk ionic concentration $\mathrm{n}_{0}$, the variation of the ionic concentration will alter the EDL thickness. In this analysis, the concentration of the two conducting fluids is in the range of $10^{-6}-10^{-5} \mathrm{M}$, therefore, the bulk concentration $n_{0}=6.022 \times 10^{20}-6.022 \times 10^{21} \mathrm{~m}^{-3}$ and the EDL dimension parameter $K=87-275$.

\subsection{Electrical potential}

Fig. 3 shows the potential distribution along the symmetrical line when the interface zeta potential $\bar{\xi}_{3}=\bar{\xi}_{6}=\bar{\xi}_{\text {wall }}$. The EDL only exists in the two conducting fluids. The interface zeta potential induces an EDL at the liquid-liquid interface, where the electroosmotic body forces are present. The result shows that the interface zeta potential affects the electrical potential distribution dramatically. The EDL profiles are shown in Fig. 3 where $K=87(1 / \kappa \approx 300 \mathrm{~nm})$ and $K=275(1 / \kappa \approx 97 \mathrm{~nm})$. It shows that the value of $K$ controls the dimensionless EDL thickness: a larger value of $\mathrm{K}$ corresponds to a thinner EDL.

Points A, B, C are indicated in Fig. 3. Point A is within the EDL at $\bar{y}=0.9925$, point $\mathrm{B}$ corresponds to the flow of conducting fluid 1 , outside the EDL at $\bar{y}=0.85$, point $C$ is in the nonconducting fluid region. The dynamic response of the flow in points $\mathrm{A}, \mathrm{B}$ and $\mathrm{C}$ will be discussed.

From Eq. (28), the velocity $\bar{u}$ of three-fluid can be decomposed into two parts, $\bar{u}^{p}$ and $\bar{u}^{E}$. $\bar{u}^{p}$ corresponds to velocity driven by pressure gradient and $\bar{u}^{E}$ corresponds to velocity driven by electroosmotic effect. With the proposed analytical model, we investigate the following cases. (1) With zero pressure gradientis applied across the microchannel, the flow is simply a three-fluid 
electroosmotic flow with the flow velocity $\bar{u}^{E}$. (2) When both the pressure gradient and the electric field are applied across the microchannel, the three-fluid is driven by the combined electroosmotic force and pressure gradient with the flow velocity $\bar{u}$.

\subsection{Three -fluid electroosmotic flow}

Figs. 4 and 5 show the time evolution of the velocity profiles, $\bar{u}^{E}$ at the symmetric line when the flow parameters are zero pressuregradient, $E_{x 1}=E_{x 3}=3000 \mathrm{~V} / \mathrm{m}, K=130, \alpha_{1}=\alpha_{2}=\alpha_{3}=1$, $\beta_{1}=\beta_{2}=\beta_{3}=1,\left(\bar{\xi}_{\text {interface }}=\bar{\xi}_{\text {wall }}\right.$ or $\left.\bar{\xi}_{\text {interface }}=0\right)$. The viscosity and density of the $\mathrm{NaCl}$ solution is $\mu_{\text {ref }}=10^{-3} \mathrm{~Pa} \mathrm{~s}$ and $\rho_{\text {ref }}=10^{3} \mathrm{~kg} / \mathrm{m}^{3}$, respectively. With these reference potential and viscosity, the Helmholtz-Smoluchowski electroosmotic velocity is chosen as the reference velocity $U_{\text {ref }}=$ $\left(E_{x 1} \varepsilon \xi_{\text {ref }}\right) / \mu_{\text {ref }}=1.59 \times 10^{-4} \mathrm{~m} / \mathrm{s}$. The corresponding Reynolds number is $\operatorname{Re} \approx 0.0063$.

Fig. 4 shows the time evolution of the velocity profile when the interface zeta potential $\bar{\xi}_{3}=\bar{\xi}_{6}=$ $\bar{\xi}_{\text {wall }}$. It can be seen that upon the application of the electric fields, the conducting fluids are activated in regions close to the channel walls and the interfaces. The velocities of the two conducting fluids increase rapidly from zero in the same direction within the EDL regions close to the walls and the interfaces. But the effects of the force from the free charges of the interface cause the interface velocity increases rapidly in the opposite direction. This is because the interface charges are equal to the total net charges within the EDL regions, close to the interface. The velocity at the interface region is significantly influenced by the surface force during the initial transient state. As time elapses, the liquid inside the EDLs exerts hydrodynamic shear stress on its adjacent liquid; the liquid outside the EDL regions may be considered as 'passive' flow caused by shear viscous forces.

For non-conducting fluid, during the initial transient time, it moves in the opposite direction. As time elapse, because the EDLs exert the hydrodynamic shear stress on the adjacent conducting fluids, the negative velocity reduces. The characteristic of non-conducting liquid depends on the matching conditions between the three liquids, which involve the external electric fields, the viscosity ratio of liquids, the interface phenomenon, and the charge density in the conducting liquids.

Fig. 5 shows the velocity profile when the interface zeta potential $\bar{\xi}_{3}=\bar{\xi}_{6}=0$. Comparing Figs. 4 with 5 , we find that: the velocity profile is completely different in the absence of the surface charge. Upon the application of the electric field, the flow is activated only in the EDL regions near the channel walls in the conducting liquids. In the non-conducting liquid, because of the absence of EDLs, there is no opposite force exerted on the interface; the non-conducting liquid is driven by the hydrodynamic viscous forces at the interfaces only. These figures show that a lower interface zeta potential would result in a higher velocity of the non-conducting liquid.

Fig. 6 shows the steady state $\left(\bar{t}>10^{-2}\right)$ dimensionless velocity profiles, $\bar{u}^{E}$, at the symmetric line, when zero pressure gradient, $E_{x 1}=E_{x 3}=3000 \mathrm{~V} / \mathrm{m}, \beta_{1}=\beta_{3}=1$ and different viscosity ratios $\beta_{2}=1,2,3$. When electric fields are applied across the conducting fluids, the conducting fluids 1 and 3 are driven by electroosmosis, which drags the non-conducting fluid 2 by the hydrodynamic 
shear force. The results indicate that the velocity profiles of the conducting fluids are strongly dependent on the viscosity ratio, $\beta_{2}$. The flow resistance of the non-conducting fluid decreases with the decreasing in $\beta_{2}$. Thus, the non-conducting fluid can be driven with less flow resistance as shown in Fig. 6. When the viscosity ratio is higher, the flow resistance of the non-conducting fluid is higher, resulting in a steeper velocity gradient at the interface of the conducting fluids.

For an extreme case, the infinite viscosities of $\mu_{2}$ and $\mu_{3}$ make the flows of the non-conducting fluid 2 and the conducting fluid 3 resemble that of the channel wall. Hence the flow of the conducting fluid 1 resembles the single-fluid EOF, through which we can compare the results of the proposed analytical model with those of the previous works. If the channel width in much larger than the channel height, in the fully developed state, the velocity profile at the symmetric section in this channel is identical with flow between two infinite parallel flat plates.

The comparison between theoretical analysis and the published two-fluid experimental data [32] is shown in Fig. 7. To simulate the flow, a infinite viscosity of $\bar{\mu}_{3}$, is assumed which make the conducting fluid 3 resemble that of the channel wall. Hence we can compare our transient model when $\bar{t}=10^{-2}$ (steady state) with the two-fluid data. Our results agree well with the published experimental data.

\subsection{Three-fluid flow driven by electroosmosis and pressure gradient}

When both pressure gradient and electric field are applied, the three liquids are driven by electroosmotic body force and pressure gradient. For a given pressure gradient, the velocities and flow rates of the three liquids depend only on the applied electroosmotic force.

Figs. 8 and 9 show the time evolution of the velocity profiles $\left(\bar{u}, \bar{u}^{p}\right.$ and $\left.\bar{u}^{E}\right)$ when $K=130, E_{x 1}$ $=E_{x 3}=3000 \mathrm{~V} / \mathrm{m}, d \bar{p} / d \bar{z}=50,000, \alpha_{1}=\alpha_{2}=\alpha_{3}=1, \beta_{1}=\beta_{2}=\beta_{3}=1$. The interface zeta potential of Figs. 8 and 9 is $\bar{\xi}_{3}=\bar{\xi}_{6}=\bar{\xi}_{\text {wall }}$ and $\bar{\xi}_{3}=\bar{\xi}_{6}=0$, respectively. When time is 0 , the fluid velocity anywhere in the channel is at rest and hence the velocity equals zero. It can be seem that upon the application of the electric field and pressure gradient, the flow begins close to the channel walls and the liquid-liquid interface, the velocity increases rapidly at the wall and interface to its peak value within the EDL regions. This reveals the unique feature of the electroosmotic flow as the flow is driven by the electrical body force due to the interaction of the applied electric field and the net charge density, such driving force occurs only within the EDL regions. The velocity profile of non-conducting liquid depends on the interface shear stress and the charge density in the conducting liquids. During this early stage of the evolution, electroosmosiseffect dominate, the velocity profile of $\bar{u}$ and $\bar{u}^{E}$ are similar as shown in Figs. 8 and 9(a).

At the steady state, the velocity profile $\bar{u}^{E}$ exhibits a plateau, resembling a plug-like flow pattern in the conducting fluids. The velocity profiles of $\bar{u}^{p}$ exhibits a parabolic profile due to the presence of the pressure gradient. The combined velocity profile, $\bar{u}$ is the superposition of the solutions of $\bar{u}^{E}$ and $\bar{u}^{p}$.

To study the dynamic responses of the flow under the applications of both constant electric field and pressure gradient, flow velocity at locations within the EDL region and outside the EDL 
are analyzed. Fig. 10(a) shows the time-dependent velocity response within the EDL at $\bar{y}=0.9925$ (point A in Fig. 3), whereas Fig. 10(b) shows the velocity response outside the EDL at $\bar{y}=0.85$ (point B in Fig. 3), and Fig. 10(c) shows the velocity response of the non-conducting fluid region at $\bar{y}=0.5$ (point $\mathrm{C}$ in Fig. 3) The flow conditions are: $K=130, E_{x 1}=E_{x 3}=3000 \mathrm{~V} / \mathrm{m}, d \bar{p} / d \bar{z}=50$, $000, \alpha_{1}=\alpha_{2}=\alpha_{3}=1, \beta_{1}=\beta_{2}=\beta_{3}=1, \bar{\xi}_{3}=\bar{\xi}_{6}=\bar{\xi}_{\text {wall }}$.

The local velocity, $\bar{u}^{\mathrm{E}}, \bar{u}^{p}$ at points $\mathrm{A}, \mathrm{B}$ and $\mathrm{C}$ are normalized by defining dimensionless variables as

$$
\begin{aligned}
& \bar{u}^{E^{*}}=\bar{u}^{E} / \bar{u}_{\text {steady state }}^{E} \\
& \bar{u}^{p^{*}}=\bar{u}^{p} / \bar{u}_{\text {steady state }}^{p}
\end{aligned}
$$

where $\bar{u}=u / U_{\text {ref}}$, and $\bar{u}_{\text {steady state }}=u_{\text {local steady state velocity }} / U_{\text {ref }}$ is the local steady state velocity.

It can be noted from Fig. 10(a) that, within the EDL region (point A), the velocity response due to pressure gradient is always behind that applied electric field. Though near the wall, the velocity is affected by the no-slip boundary condition, however, within the EDL region the flow is driven by the electrical body force resulting from the interaction of the electric field and the net charge density. As time elapse, the liquid inside the EDL exerts viscous shear stress to the adjacent liquid and fluids outsides the EDL (point B) is set in motion layer by layer, finally extending to the entire region of the conducting liquid, in this region, electroosmosis effect dominate.

At the bulk conducting liquid region (region away from EDL, at point B), the liquid is driven by the viscous shear diffusion as the result of (i) the faster fluid layers at the EDL region and (ii) the faster lamina fluid layers immediately closer to the center, i.e. the combined electric field and pressure gradient which pull the fluid at the bulk conducting liquid region. The results show that both $\bar{u}^{E}$ and $\bar{u}^{p}$ achieve the steady state at $\bar{t}=10^{-5}$.

Fig. 10(c) shows the flow in the non-conducting fluid region. The velocity response due to the pressure gradient is faster that of the applied electric field, pressure gradient effect dominates. The liquid at point $\mathrm{C}$ is driven by: (i) the faster lamina immediately closer to the center due to pressure gradient, and (ii) the interfacial shear stress. Same as before, both $\bar{u}^{E}$ and $\bar{u}^{p}$ achieve the steady state at $\bar{t}=10^{-5}$.

\subsection{Volumetric flow rate}

The dimensionless volumetric flow rates of the three-fluid electroosmotic flow, $\bar{q}_{1}, \bar{q}_{2}$ and $\bar{q}_{3}$, where zero gradient $(d \bar{p} / d \bar{z}=0), E_{x 1}=E_{x 3}=3000 \mathrm{~V} / \mathrm{m}, K=130, \rho_{1}=\rho_{2}=\rho_{3}$, are plotted in Fig. 11 for different values of interface zeta potentials (Fig. 11(a)), dynamic viscosity ratios $\beta_{2}$ (Fig. 11(b)), and kinematics viscosity ratio $\alpha_{2}$ (Fig. 11(c)). 
In Fig. 11(a), the dynamic viscosity and density of three liquids are identical. It can be seen that the higher interface zeta potentials will induce a large flow rate of the conducting liquids $\left(\bar{q}_{1}\right.$ and $\left.\bar{q}_{3}\right)$, but the flow rate of the non-conducting liquid $\left(\bar{q}_{2}\right)$ is lower. In the presence of free charges at the interface, the non-conducting liquid will flow in the opposite direction. Gao [24] called this phenomena "back flow". The back flow rate increases as the increase of the interface zeta potential. As shown in Fig. 11(a), if the interface zeta potentials are larger than the wall zeta potential, the flow rate of the non-conducting liquid may be negative at the steady state.

Fig. 11(b) shows the effect of different viscosity ratio, $\beta_{2}$., on the volumetric flow rate. In this figure, $\xi_{3}=\xi_{6}=0.5 \xi_{1}, \rho_{1}=\rho_{2}=\rho_{3}, \beta_{1}=\beta_{3}=1$. The results show that the liquid with a lower viscosity ratio can be driven with a lower flow resistance, in another word; the volumetric flow rate is higher under the same electric fields. Hence the three-liquid electroosmotic pumping method is feasible for the relatively small interface zeta potentials and viscosity ratio $\beta_{2}$.

Fig. 11(c) shows the volumetric flow rates for different density of the non-conducting liquid. In this figure, $\xi_{3}=\xi_{6}=0.5 \xi_{1}, \beta_{1}=\beta_{2}=\beta_{3}=1, \rho_{1}=\rho_{3}$. The results show that the density does not affect the velocity profile but affects the developing time, this figure shows that the nonconducting liquid with a higher density requires a longer developing time to approach the steady state. The kinematics viscosity ratio, $\alpha_{2}=\left(\bar{\mu}_{2} / \bar{\rho}_{2}\right) /\left(\bar{\mu}_{1} / \bar{\rho}_{1}\right)$, evaluates the developing time.

Fig. 12 shows the dimensionless volumetric flow rates of the three-fluid flow for case (a) $E_{x 1}=$ $E_{x 3}=3000 \mathrm{~V} / \mathrm{m}, d \bar{p} / d \overline{\mathrm{z}}=0$, and case (b) $E_{x 1}=E_{x 3}=3000 \mathrm{~V} / \mathrm{m}, d \bar{p} / d \overline{\mathrm{z}}=10,000$. As shown, the effect of pressure gradient does not affect the developing time. Volumetric flow rate is higher under the combined electroosmotic and pressure gradient.

\section{Conclusion}

An analysis of the transient three-liquid flow, which driven by the combined electroosmotic force and pressure gradient, is presented in this work. By considering the electroosmotic force as the body forces in the conducting liquids, this paper solves the linear Poisson-Boltzmann equation and Navier-Stokes equation together by the analytical method. Due to the presence of the surface free charge densities at the liquid-liquid interface, the forces in the diffuse layers and the forces exerted at the interfaces are accounted in this model. The velocity profiles and the flow rates for the transient, two-dimensional three-liquid flow is obtained analytically by the method of Laplace transform method.

The computational results show that the three-liquid electroosmotic pumping is feasible for the relative small interface zeta potential and viscosity ratio $\beta_{2}$. The detail insight of the flow characteristics of this flow configuration are provided by the velocity profile as time evolution.

\section{Appendix A}

In the following, we will define several auxiliary functions which facilitate the analytical evaluation of the pertinent expressions in the present work. All these functions are obtained through integrating matching conditions, velocity profiles and shear stress at the interface. They are defined as follows: 


$$
\begin{aligned}
& A=\tanh \left[D_{1 j}\left(\bar{h}+\bar{h}_{1}\right)\right] \cosh \left(D_{1 j} \bar{h}\right) \sinh \left(E_{2 j} \bar{h}\right)-\sinh \left(D_{1 j} \bar{h}\right) \\
& \times \sinh \left(E_{2 j} \bar{h}\right)-\frac{D_{1 j}}{\beta_{1} E_{2 j}} \tanh \left[D_{1 j}\left(\bar{h}+\bar{h}_{1}\right)\right] \sinh \left(D_{1 j} \bar{h}\right) \\
& \times \cosh \left(E_{2 j} \bar{h}\right)+\frac{D_{1 j}}{\beta_{1} E_{2 j}} \cosh \left(D_{1 j} \bar{h}\right) \cosh \left(E_{2 j} \bar{h}\right) \\
& B=-\frac{E_{3 j}}{\left(\beta_{1} / \beta_{3}\right) E_{2 j}} \tanh \left[E_{3 j}\left(\bar{h}+\bar{h}_{3}\right)\right] \sinh \left(E_{3 j} \bar{h}\right) \cosh \left(E_{2 j} \bar{h}\right) \\
& +\frac{E_{3 j}}{\left(\beta_{1} / \beta_{3}\right) E_{2 j}} \cosh \left(E_{3 j} \bar{h}\right) \cosh \left(E_{2 j} \bar{h}\right)+\tanh \left[E_{3 j}\left(\bar{h}+\bar{h}_{3}\right)\right] \\
& \times \cosh \left(E_{3 j} \bar{h}\right) \sinh \left(E_{2 j} \bar{h}\right)-\sinh \left(E_{3 j} \bar{h}\right) \sinh \left(E_{2 j} \bar{h}\right) \\
& C=-\frac{\sinh \left(E_{3 j} \bar{h}\right)}{2 \cosh \left[E_{3 j}\left(\bar{h}+\bar{h}_{3}\right)\right] \sinh \left(E_{2 j} \bar{h}\right)} \\
& D=-\frac{\sinh \left(D_{1 j} \bar{h}\right)}{2 \cosh \left[D_{1 j}\left(\bar{h}+\bar{h}_{1}\right)\right] \sinh \left(E_{2 j} \bar{h}\right)} \\
& G=-\frac{\phi_{j}\left(\bar{h}+\bar{h}_{3}, s\right) \cosh \left(E_{3 j} \bar{h}\right)}{2 \sinh \left(E_{2 j} \bar{h}\right) \cosh \left[E_{3 j}\left(\bar{h}+\bar{h}_{3}\right)\right]}+\frac{\phi_{3 j}(\bar{h}, s)-\phi_{1 j}(-\bar{h}, s)}{2 \sinh \left(E_{2 j} \bar{h}\right)} \\
& +\frac{\phi_{j}\left(-\bar{h}-\bar{h}_{1}, s\right) \cosh \left(D_{1 j} \bar{h}\right)}{2 \sinh \left(E_{2 j} \bar{h}\right) \cosh \left[D_{1 j}\left(\bar{h}+\bar{h}_{1}\right)\right]} \\
& H=\frac{\phi_{1 j}\left(-\bar{h}-\bar{h}_{1}, s\right)}{A \cosh \left[D_{1 j}\left(\bar{h}+\bar{h}_{1}\right)\right]} \sinh \left(E_{2 j} \bar{h}\right) \cosh \left(D_{1 j} \bar{h}\right) \\
& -\frac{\sinh \left(E_{2 j} \bar{h}\right) \phi_{1 j}(-\bar{h}, s)}{A}-\frac{M_{1} \bar{\rho}_{q 1}^{s}(\bar{z}) \cosh \left(E_{2 j} \bar{h}\right)}{A \beta_{1} E_{2 j}} \\
& -\frac{\phi_{1 j}^{\prime}(-\bar{h}, s) \cosh \left(E_{2 j} \bar{h}\right)}{A \beta_{1} E_{2 j}}-\frac{D_{1 j}}{A \beta_{1} E_{2 j}} \frac{\phi_{1 j}\left(-\bar{h}-\bar{h}_{1}, s\right)}{\cosh \left[D_{1 j}\left(\bar{h}+\bar{h}_{1}\right)\right]} \\
& \times \sinh \left(D_{1 j} \bar{h}\right) \cosh \left(E_{2 j} \bar{h}\right) \\
& I=\frac{E_{3 j} \cosh \left(E_{2 j} \bar{h}\right)}{B\left(\beta_{1} / \beta_{3}\right) E_{2 j}} \frac{\phi_{3 j}\left(\bar{h}+\bar{h}_{3}, s\right)}{\cosh \left[E_{3 j}\left(\bar{h}+\bar{h}_{3}\right)\right]} \sinh \left(E_{3 j} \bar{h}\right) \\
& -\frac{\phi_{3 j}^{\prime}(\bar{h}, s) \cosh \left(E_{2 j} \bar{h}\right)}{B\left(\beta_{1} / \beta_{3}\right) E_{2 j}}-\frac{M_{3} \bar{\rho}_{q 3}^{s}(\bar{z}) \cosh \left(E_{2 j} \bar{h}\right)}{B\left(\beta_{1} / \beta_{3}\right) E_{2 j}} \\
& +\frac{\sinh \left(E_{2 j} \bar{h}\right) \phi_{3 j}(\bar{h}, s)}{B}-\frac{\phi_{3 j}\left(\bar{h}+\bar{h}_{3}, s\right)}{B \cosh \left[E_{3 j}\left(\bar{h}+\bar{h}_{3}\right)\right]} \sinh \left(E_{2 j} \bar{h}\right) \\
& \times \cosh \left(E_{3 j} \bar{h}\right)
\end{aligned}
$$




$$
\begin{aligned}
& J=\frac{\tanh \left[D_{1 j}\left(\bar{h}+\bar{h}_{1}\right)\right] \cosh \left(D_{1 j} \bar{h}\right)-\sinh \left(D_{1 j} \bar{h}\right)}{2 \cosh \left(E_{2 j} \bar{h}\right)} \\
& K=\frac{-\tanh \left[E_{3 j}\left(\bar{h}+\bar{h}_{3}\right)\right] \cosh \left(E_{3 j} \bar{h}\right)+\sinh \left(E_{3 j} \bar{h}\right)}{2 \cosh \left(E_{2 j} \bar{h}\right)} \\
& L=-\frac{\phi_{3 j}\left(\bar{h}+\bar{h}_{3}, s\right) \cosh \left(E_{3 j} \bar{h}\right)}{2 \cosh \left(E_{2 j} \bar{h}\right) \cosh \left[E_{3 j}\left(\bar{h}^{\prime}+\bar{h}_{3}\right)\right]}+\frac{\phi_{3 j}(\bar{h}, s)+\phi_{1 j}(-\bar{h}, s)}{2 \cosh \left(E_{2 j} \bar{h}\right)} \\
& -\frac{\phi_{1 j}\left(-\bar{h}-\bar{h}_{1}, s\right) \cosh \left(D_{1 j} \bar{h}\right)}{2 \cosh \left(E_{2 j} \bar{h}\right) \cosh \left[D_{1 j}\left(\bar{h}+\bar{h}_{1}\right)\right]} \\
& M=-\frac{\operatorname{Re}}{S \lambda_{j}} \frac{d \bar{P}}{d \bar{x}} \sqrt{\frac{2}{\bar{w}}}(-1)^{j} \\
& \times\left\{\frac{1}{2 E_{3 j}^{2} \beta_{3} \cosh \left(E_{2 j} \bar{h}\right)}+\frac{1}{2 D_{1 j}^{2} \cosh \left(E_{2 j} \bar{h}\right)}-\frac{1}{\beta_{2} E_{2 j}^{2} \cosh \left(E_{2 j} \bar{h}\right)}\right\} \\
& +\frac{\operatorname{Re}}{\lambda_{j} s} \frac{d \bar{P}}{d \bar{x}} \sqrt{\frac{2}{\bar{w}}}(-1)^{j}\left\{\frac{\cosh \left(E_{3 j} \bar{h}\right)}{2 E_{3 j}^{2} \beta_{3} \cosh \left(E_{2 j} \bar{h}\right) \cosh \left[E_{3 j}\left(\bar{h}_{3}+\bar{h}\right)\right]}\right. \\
& \left.+\frac{\cosh \left(D_{1 j} \bar{h}\right)}{2 D_{1 j}^{2} \cosh \left(E_{2 j} \bar{h}\right) \cosh \left[D_{1 j}\left(\bar{h}_{1}+\bar{h}\right)\right]}\right\} \\
& N=-\frac{\sinh \left(E_{3 j} \bar{h}_{3}\right)}{2 \cosh \left(E_{2 j} \bar{h}\right) \cosh \left[E_{3 j}\left(\bar{h}_{3}+\bar{h}\right)\right]} \\
& O=\frac{\operatorname{Re}}{\lambda_{j} s} \frac{d \bar{P}}{d \bar{x}} \sqrt{\frac{2}{\bar{w}}}(-1)^{j} \\
& \times\left\{\frac{\cosh \left(E_{3 j} \bar{h}\right)}{2 E_{3 j}^{2} \beta_{3} \sinh \left(E_{2 j} \bar{h}\right) \cosh \left[E_{3 j}\left(\bar{h}_{3}+\bar{h}\right)\right]}+\frac{1}{2 D_{1 j}^{2} \sinh \left(E_{2 j} \bar{h}\right)}\right\} \\
& -\frac{\operatorname{Re}}{S \lambda_{j}} \frac{d \bar{P}}{d \bar{x}} \sqrt{\frac{2}{\bar{w}}}(-1)^{j}\left\{\frac{\cosh \left(D_{1 j} \bar{h}\right)}{2 D_{1 j}^{2} \sinh \left(E_{2 j} \bar{h}\right) \cosh \left[D_{1 j}\left(\bar{h}_{1}+\bar{h}\right)\right]}+\frac{1}{2 E_{3 j}^{2} \beta_{3} \sinh \left(E_{2 j} \bar{h}\right)}\right\} \\
& P=-\frac{\sinh \left(E_{3 j} \bar{h}_{3}\right)}{2 \sinh \left(E_{2 j} \bar{h}\right) \cosh \left[E_{3 j}\left(\bar{h}_{3}+\bar{h}\right)\right]} \\
& Q=\frac{\sinh \left(D_{1 j} \bar{h}_{1}\right)}{2 \cosh \left(E_{2 j} \bar{h}\right) \cosh \left[D_{1 j}\left(\bar{h}_{1}+\bar{h}\right)\right]}
\end{aligned}
$$




$$
\begin{aligned}
& R=-\frac{\sinh \left(D_{1 j} \bar{h}_{1}\right)}{2 \sinh \left(E_{2 j} \bar{h}\right) \cosh \left[D_{1 j}\left(\bar{h}_{1}+\bar{h}\right)\right]} \\
& S=\frac{\sinh \left(D_{1 j} \bar{h}_{1}\right) \sinh \left(E_{2 j} \bar{h}\right)}{\cosh \left[D_{1 j}\left(\bar{h}_{1}+\bar{h}\right)\right]}+\frac{D_{1 j} \cosh \left(D_{1 j} \bar{h}_{1}\right) \cosh \left(E_{2 j} \bar{h}\right)}{\beta_{2} E_{2 j} \cosh \left[D_{1 j}\left(\bar{h}_{1}+\bar{h}\right)\right]} \\
& T=\frac{\operatorname{Re}}{S \lambda_{j} D_{1 j}^{2}} \frac{d \bar{P}}{d \bar{x}} \sqrt{\frac{2}{\bar{w}}}(-1)^{j}\left\{\frac{\cosh \left(D_{1 j} \bar{h}\right) \sinh \left(E_{2 j} \bar{h}\right)}{\cosh \left[D_{1 j}\left(\bar{h}_{1}+\bar{h}\right)\right]}-\sinh \left(E_{2 j} \bar{h}\right)\right\} \\
& -\frac{\operatorname{Re}}{s \beta_{2} \lambda_{j} E_{2 j}} \frac{d \bar{P}}{d \bar{x}} \sqrt{\frac{2}{\bar{w}}}(-1)^{j}\left\{\frac{\sinh \left(D_{1 j} \bar{h}\right) \cosh \left(E_{2 j} \bar{h}\right)}{D_{1 j} \cosh \left[D_{1 j}\left(\bar{h}_{1}+\bar{h}\right)\right]}-\frac{\sinh \left(E_{2 j} \bar{h}\right)}{s \beta_{2} \lambda_{j} E_{2 j}}\right\} \\
& V=\frac{\sinh \left(E_{2 j} \bar{h}\right) \sinh \left(E_{3 j} \bar{h}_{3}\right)}{\cosh \left[E_{3 j}\left(\bar{h}_{3}+\bar{h}\right)\right]}+\frac{E_{3 j} \cosh \left(E_{3 j} \bar{h}_{3}\right) \cosh \left(E_{2 j} \bar{h}\right)}{\left(\beta_{1} / \beta_{3}\right) E_{2 j} \cosh \left[E_{3 j}\left(\bar{h}_{3}+\bar{h}\right)\right]} \\
& U=\frac{1}{E_{3 j}} \frac{\operatorname{Re}}{\lambda_{j} s \beta_{1} E_{2 j}} \frac{d \bar{P}}{d \bar{x}} \sqrt{\frac{2}{\bar{w}}}(-1)^{j} \frac{\sinh \left(E_{3 j} \bar{h}\right) \cosh \left(E_{2 j} \bar{h}\right)}{\cosh \left[E_{3 j}\left(\bar{h}_{3}+\bar{h}\right)\right]}-\operatorname{Re} \\
& \times \frac{d \bar{P}}{d \bar{x}} \times \sqrt{\frac{2}{\bar{w}}} \times \frac{(-1)^{j} \sinh \left(E_{2 j} \bar{h}\right)}{s \beta_{2} \lambda_{j} E_{2 j}^{2}}-\frac{1}{E_{3 j}^{2}} \frac{\operatorname{Re}}{\lambda_{j} s \beta_{3}} \frac{d \bar{P}}{d \bar{x}} \sqrt{\frac{2}{\bar{w}}} \\
& \times(-1)^{j}\left\{\frac{\cosh \left(E_{3 j} \bar{h}\right) \sinh \left(E_{2 j} \bar{h}\right)}{\cosh \left[E_{3 j}\left(\bar{h}_{3}+\bar{h}\right)\right]}-\sinh \left(E_{2 j} \bar{h}\right)\right\} \\
& \phi_{1 j}(\bar{y}, s)=\sqrt{\frac{\bar{w}}{2}} \frac{4 \times \operatorname{Re} \times G_{x 1} \times(-1)^{j+1}}{(2 j+1) s \pi \sinh \left[B_{j} h_{1}\right]} \\
& \times\left\{\bar{\xi}_{1} \frac{\sinh \left[B_{j}(\bar{h}+\bar{y})\right]}{\left(B_{j}^{2}-D_{1 j}^{2}\right)}-\bar{\xi}_{3} \frac{\sinh \left[B_{j}\left(h_{1}+\bar{h}+\bar{y}\right)\right]}{\left(B_{j}^{2}-D_{1 j}^{2}\right)}\right\} \\
& +\sqrt{\frac{2}{\bar{w}}} \sum_{p=1}^{\infty} \frac{2 \times \operatorname{Re} \times G_{x 1} \times \bar{\xi}_{2} \times \lambda_{j}}{p \pi s\left(A_{p}^{2}+\lambda_{j}^{2}\right)}\left[(-1)^{p}-1\right] \\
& \times(-1)^{j+1} \frac{\sin \left[\frac{p \pi}{h_{1}}(\bar{h}+\bar{y})\right]}{\left[\left(\frac{p p i}{h_{1}}\right)^{2}+D_{1 j}^{2}\right]}
\end{aligned}
$$




$$
\begin{aligned}
& \phi_{3 j}(\bar{y}, s)=\sum_{p=1}^{\infty} \frac{4 \bar{\xi}_{2}(-1)^{j+1} \lambda_{j}}{p \pi \bar{w} s\left(A_{p 3}^{2}+\lambda_{j}^{2}\right)\left[\left(\frac{p \pi}{h_{3}}\right)^{2}+\lambda_{j}^{2}+s\right]} \times\left[(-1)^{p+1}+1\right] \\
& \times \sin \left[\frac{p}{\bar{h}_{3}} \pi(\bar{y}-\bar{h})\right]+\sum_{j=1}^{\infty} \frac{4 \operatorname{Re} G_{x 3}(-1)^{j+1}}{(2 j-1) s \pi \beta_{3} \sinh \left[B_{j} \bar{h}_{3}\right]\left(B_{j}^{2}-\lambda_{j}^{2}-s\right)} \\
& \times\left\{\bar{\xi}_{4} \sinh \left[B_{j}(\bar{y}-\bar{h})\right]-\bar{\xi}_{6} \sinh \left[B_{j}\left(\bar{y}-\bar{h}_{3}-\bar{h}\right)\right]\right\} \\
& \bar{Q}_{1}^{E}(s)=\sum_{j=0}^{\infty} \frac{2 \sin \left(\lambda_{j} \bar{w}\right)}{\lambda_{j}}\left\{\frac{1}{D_{1 j}} \times \tanh \left[D_{1 j}\left(\bar{h}+\bar{h}_{1}\right)\right] \times\left[\sinh \left(D_{1 j} \bar{h}\right)\right.\right. \\
& \left.\left.-\sinh \left(D_{1 j}\left(\bar{h}+\bar{h}_{1}\right)\right)\right]+\frac{1}{D_{1 j}}\left[\cosh \left(D_{1 j}\left(\bar{h}+\bar{h}_{1}\right)\right)+\cosh \left(D_{1 j} \bar{h}\right)\right]\right\} b_{1 j}^{E} \\
& +\sum_{j=0}^{\infty} \frac{2 \sin \left(\lambda_{j} \bar{w}\right)}{\lambda_{j}}\left\{\phi_{1 j}^{I}\left(-\bar{h}-\bar{h}_{1}, s\right)-\phi_{1 j}^{I}(-\bar{h}, s)\right. \\
& \left.+\frac{1}{D_{1 j}} \frac{\phi_{1 j}\left(-\bar{h}-\bar{h}_{1}, s\right)}{\cosh \left[D_{1 j}\left(\bar{h}+\bar{h}_{1}\right)\right]}\left[\sinh \left(D_{1 j} \bar{h}\right)-\sinh \left(D_{1 j}\left(\bar{h}+\bar{h}_{1}\right)\right)\right]\right\} \\
& \bar{Q}_{1}^{p}(s)=\sum_{j=0}^{\infty} \frac{2}{\lambda_{j}} \sin \left(\lambda_{j} \bar{w}\right) \frac{\operatorname{Re}}{s D_{1 j}^{2}} \frac{d \bar{P}}{d \bar{x}} \frac{4 \times(-1)^{j+1}}{(2 j-1) \pi} \frac{1}{D_{1 j}} \\
& \times\left\{\frac{\sinh \left(D_{1 j} \bar{h}\right)-\sinh \left(D_{1 j}\left(\bar{h}+\bar{h}_{1}\right)\right)}{\cosh \left[D_{1 j}\left(\bar{h}_{1}+\bar{h}\right)\right]}+\bar{h}_{1}\right\}+\sum_{j=0}^{\infty} \frac{2}{\lambda_{j}} \sin \left(\lambda_{j} \bar{w}\right) \\
& \times \frac{\operatorname{Re}}{s D_{1 j}^{2}} \frac{d \bar{P}}{d \bar{x}} \frac{4 \times(-1)^{j+1}}{(2 j-1) \pi} \frac{1}{D_{1 j}} \times\left\{\operatorname { t a n h } [ D _ { 1 j } ( \overline { h } _ { 1 } + \overline { h } ) ] \left[\sinh \left(D_{1 j} \bar{h}\right)\right.\right. \\
& \left.\left.-\sinh \left(D_{1 j}\left(\bar{h}+\bar{h}_{1}\right)\right)\right]+\left[\cosh \left(D_{1 j}\left(\bar{h}+\bar{h}_{1}\right)\right)-\cosh \left(D_{1 j} \bar{h}\right)\right]\right\} b_{1 j}^{P} \\
& \bar{Q}_{2}^{E}(s)=4 \sum_{j=0}^{\infty} \frac{a_{2 j}^{E} \sinh \left(E_{2 j} \bar{h}\right) \sin \left(\lambda_{j} \bar{w}\right)}{\lambda_{j} E_{2 j}} \\
& \bar{Q}_{2}^{p}(s)=4 \sum_{j=0}^{\infty}\left[a_{2 j}^{p} \sinh \left(E_{2 j} \bar{h}\right)-\frac{1}{E_{2 j}^{2}} \frac{\operatorname{Re}}{s \beta_{2}} \frac{4 \times(-1)^{j+1}}{(2 j-1) \pi} \bar{h}\right] \frac{\sin \left(\lambda_{j} \bar{w}\right)}{\lambda_{j} E_{2 j}} \\
& \bar{Q}_{3}^{E}(s)=2 \sum_{j=0}^{\infty}\left\{\frac { 1 } { E _ { 3 j } } \left\{-\tanh \left[E_{3 j}\left(\bar{h}+\bar{h}_{3}\right)\right]\left[\sinh \left(E_{3 j}\left(\bar{h}+\bar{h}_{3}\right)\right)-\sinh \left(E_{3 j} \bar{h}\right)\right]\right.\right. \\
& \left.+\cosh \left(E_{3 j}\left(\bar{h}+\bar{h}_{3}\right)\right)-\cosh \left(E_{3 j} \bar{h}\right)\right\} b_{3 j}^{E}+2 \sum_{j=0}^{\infty} \frac{\sin \left(\lambda_{j} \bar{w}\right)}{\lambda_{j}} \\
& \times\left\{\phi_{3 j}^{I}\left(\bar{h}+\bar{h}_{3}, s\right)-\phi_{3 j}^{I}(\bar{h}, s)-\frac{1}{E_{3 j}} \frac{\phi_{3 j}\left(\bar{h}+\bar{h}_{3}, s\right)}{\cosh \left[E_{3 j}\left(\bar{h}+\bar{h}_{3}\right)\right]}\left[\sinh \left(E_{3 j}\left(\bar{h}+\bar{h}_{3}\right)\right)\right.\right. \\
& \left.\left.-\sinh \left(E_{3 j} \bar{h}\right)\right]\right\}
\end{aligned}
$$




$$
\begin{aligned}
\bar{Q}_{3}^{p}(s)= & 2 \sum_{j=0}^{\infty}\left\{\frac{\sin \left(\lambda_{j} \bar{z}\right)}{\lambda_{j} E_{3 j}} \frac{1}{E_{3 j}^{2}} \frac{\operatorname{Re}}{s \beta_{3}} \frac{d \bar{P}}{d \bar{x}} \frac{4 \times(-1)^{j+1}}{(2 j-1) \pi}\right. \\
& \times\left\{\frac{1}{E_{3 j}} \frac{\sinh \left(E_{3 j}\left(\bar{h}+\bar{h}_{3}\right)\right)-\sinh \left(E_{3 j} \bar{h}\right)}{\cosh \left[E_{3 j}\left(\bar{h}_{3}+\bar{h}\right)\right]}-\bar{h}_{3}\right\} \\
& +\left\{-\tanh \left[E_{3 j}\left(\bar{h}_{3}+\bar{h}\right)\right]\left[\sinh \left(E_{3 j}\left(\bar{h}+\bar{h}_{3}\right)\right)-\sinh \left(E_{3 j} \bar{h}\right)\right]\right. \\
& \left.\left.+\left[\cosh \left(E_{3 j}\left(\bar{h}+\bar{h}_{3}\right)\right)-\cosh \left(E_{3 j} \bar{h}\right)\right]\right\} b_{3 j}^{P}\right\}
\end{aligned}
$$




\section{References}

[1] B.H Kunst, A, Schots, A.J.W.G. Visser, Design of a confocal microfluidic particle sorter using fluorescent photon burst detection,Rev.Sci.Instrum.75 (2004)2892-2898.

[2] J.Clemmens, H. Hess, R. Doot, C.M.Matzke, G.D.Bachand, V.Vogel ,Motorprotein roundabouts: microtubulesmoving on kinesin-coated tracks through engineered networks, Lab Chip - Miniaturisation Chem.Biol.4(2004)83-86.

[3] F.Petersson , A.Nilsson, C.Holm,H.Jounsson, T.Laurell, Continuous separation of lipid particles from erythrocytes by means of laminar flow and acoustic standing wave forces,Lab Chip -MiniaturisationChem .B IOL.5(2005)20-22 .

[4] S.K Sia, V. Linder, B.A. Parviz, A. Siegel,G.M. Whitesides, An intergrated approach to a portable and low-cost immunoassay for resource-poor settings,Angew.Chem-Int.Ed. 43 (2004) 498-502.

[5] R. Riehn, M. Lu, Y.M. Wang, S.F. Lim, E.C. Cox, R.H. Austin, Restriction mapping in nanofluidic devices, Proc. Natl. Acad. Sci. USA 102 (2005) 10012-10016.

[6] A.M. Skelley, J.R. Scherer, A.D. Aubrey, W.H. Grover, R.H.C. Ivester, P. Ehrenfreund, F.J. Grunthaner, J.L. Bada, R.A. Mathies, Development and evaluation of a microdevice for amino acid biomarker detection and analysis on Mars, Proc. Natl. Acad. Sci. USA 102 (2005) 1041-1046.

[7] P.S. Dittrich, B. Müller, P. Schwille, Studying reaction kinetics by simultaneous FRET and cross-correlation analysis in a miniaturized continuous flow reactor, Phys. Chem. Chem. Phys. 6 (2004) 4416-4420.

[8] J. Wang, J. Zima, N.S. Lawrence, M.P. Chatrathi, A. Mulchandani, G.E. Collins, Microchip capillary electrophoresis with electrochemical detection of thiolcontaining degradation products of V-type nerve agents, Anal. Chem. 76 (2004) 4721-4726.

[9] C.J. Lu, W.H. Steinecker, W.C. Tian, M.C. Oborny, J.M. Nichols, M. Agah, J.A. Potkay, H.K.L. Chan, J. Driscoll, R.D. Sacks, K.D. Wise, S.W. Pang, E.T. Zellers, First-generation hybrid MEMS gas chromatograph, Lab Chip - Miniaturisation Chem. Biol. 5 (2005) 1123-1131.

[10] R. Chein, S.H. Tsai, Microfluidic flow switching design using volume of fluid model, Biomed. Microdevices 6 (2004) 81-90.

[11] Z. Wu, N.T. Nguyen, Hydrodynamic focusing in microchannels under consideration of diffusive dispersion: theories and experiments, Sens. Actuators B 107 (2005) 965-974.

[12] D. Erickson, D. Li, C. Werner, An improved method of determining the $\xi$-potential and surface conductance, J. Colloid Interface Sci. 232 (2000) 186- 197.

[13] L.M. Fu, R.J. Yang, C.H. Lin, Y.J. Pan, G.B. Lee, Electrokinetically driven micro flow cytometers with integrated fiber optics for on-line cell/particle detection, Anal. Chim. Acta 507 (2004) 163-169.

[14] A. Brask, G. Goranovic, H. Bruus, Electroosmotic pumping of nonconducting liquids by viscous drag from a secondary conducting liquid, in: 2003 Nanotechnology Conference and Trade Show - Nanotech 2003, 2003, pp. 190-193.

[15] G. Goranovic, Electrohydrodynamic Aspects of Two-fluid Microfluidic Systems: Theory and Simulation, Mikroelektronikcentret, Technical University of Denmark, 2003. 
[16] Y. Gao, T.N. Wong, C. Yang, K.T. Ooi, Two-fluid electroosmotic flow in microchannels, J. Colloid Interface Sci. 284 (2005) 306-314.

[17] Y. Gao, C. Wang, T.N. Wong, C. Yang, N.T. Nguyen, K.T. Ooi, Electro-osmotic control of the interface position of two-liquid flow through a microchannel, J. Micromech. Microeng. 17 (2007) 358-366.

[18] P. Dutta, A. Beskok, Analytical solution of combined electroosmotic/pressure driven flows in two-dimensional straight channels: finite Debye layer effects, Anal. Chem. 73 (2001) 1979-1986.

[19] J.G. Santiago, Electroosmotic flows in microchannels with finite inertial and pressure forces, Anal. Chem. 73 (2001) 2353-2365.

[20] A.Q. Zade, M.T. Manzari, S.K. Hannani, An analytical solution for thermally fully developed combined pressure - electroosmotically driven flow in microchannels, Int. J. Heat Mass Transfer 50 (2007) 1087-1096.

[21] H.J. Keh, H.C. Tseng, Transient electrokinetic flow in fine capillaries, J. Colloid Interface Sci. 242 (2001) 450-459.

[22] Marcos, K.T. Ooi, C. Yang, J.C. Chai, T.N. Wong, Developing electro-osmotic flow in closed-end micro-channels, Int. J. Eng. Sci. 43 (2005) 1349-1362.

[23] C. Yang, C.B. Ng, V. Chan, Transient analysis of electroosmotic flow in a slit microchannel, J. Colloid Interface Sci. 248 (2002) 524-527.

[24] Y. Gao, T.N. Wong, C. Yang, T.O. Kim, Transient two-liquid electroosmotic flow with electric charges at the interface, Colloids Surf. A: Physicochem. Eng. Aspects 266 (2005) 117-128.

[25] R.J. Hunter, Zeta Potential in Colloid Science: Principles and Applications, vol. 18, London, 1981.

[26] N.A. Patankar, H.H. Hu, Numerical simulation of electroosmotic flow, Anal. Chem. 70 (1998) 1870-1881.

[27] R.F. Probstein, Physicochemical Hydrodynamics: An Introduction, vol. 190, New York, 1994.

[28] J.C. Baygents, D.A. Saville, Electrophoresis of small particles and fluid globules in weak electrolytes, J. Colloid Interface Sci. 146 (1991) 9-37.

[29] D. Maillet, Thermal Quadrupoles: Solving the Heat Equation through Integral Transform, John Wiley \& Sons, 2000.

[30] Y. Gu, D. Li, An electrical suspension method for measuring the electric charge on small silicone oil droplets dispersed in aqueous solutions, J. Colloid Interface Sci. 195 (1997) 343-352.

[31] Y. Gu, D. Li, The $\xi$-potential of silicone oil droplets dispersed in aqueous solutions, J. Colloid Interface Sci. 206 (1998) 346-349.

[32] C. Wang, Y. Gao, N.T. Nguyen, T.N. Wong, C. Yang, K.T. Ooi, Interface control of pressure-driven two-fluid flow in microchannels using electroosmosis, J. Micromech. Microeng. 15 (2005) 2289-2297. 


\section{List of Figures}

Fig.1 Schematic of the phenomenon and coordinate system.

Fig.2 Schematic representation of the three-fluid electroosmotic stratified flow.

Fig.3 Potential distributions along the symmetrical line when $\bar{\xi}_{3}=\bar{\xi}_{6}=\bar{\xi}_{\text {wall }}$. Point A is within the EDL at $\bar{y}=0.9925$; Point $\mathrm{B}$ is outside the $\mathrm{EDL}$ at $\bar{y}=0.85$; Point $\mathrm{C}$ is in the non-conducting fluid region at $\bar{y}=0.5$.

Fig.4 Time evolution of the three-fluid electroosmotic flow (a) $\bar{t}=1 \times 10^{-6}$; (b) $\bar{t}=1 \times 10^{-5}$; (c) $\bar{t}=1 \times 10^{-4} ;(\mathrm{d}) \bar{t}=1 \times 10^{-2} \quad\left(K=130, E_{x 1}=E_{x 3}=3000 \mathrm{~V} / \mathrm{m}, \frac{d \bar{p}}{d \bar{z}}=0, \alpha_{1}=\alpha_{2}=\alpha_{3}=1\right.$, $\left.\beta_{1}=\beta_{2}=\beta_{3}=1, \bar{\xi}_{3}=\bar{\xi}_{1}=\bar{\xi}_{4}=\bar{\xi}_{6}=\bar{\xi}_{\text {wall }}\right)$.

Fig.5 Time evolution of the three-fluid electroosmotic flow (a) $\bar{t}=1 \times 10^{-6}$; (b) $\bar{t}=1 \times 10^{-5}$; (c) $\bar{t}=1 \times 10^{-4} ;(\mathrm{d}) \bar{t}=1 \times 10^{-2} \quad\left(K=130, E_{x 1}=E_{x 3}=3000 \mathrm{~V} / \mathrm{m}, \frac{d \bar{p}}{d \bar{z}}=0, \alpha_{1}=\alpha_{2}=\alpha_{3}=1\right.$, $\beta_{1}=\beta_{2}=\beta_{3}=1, \bar{\xi}_{3}=\bar{\xi}_{6}=0$ ).

Fig.6 Dimensionless steady state velocity distribution at the symmetric line for different values of dynamic viscosity ratio, $\beta_{2}\left(E_{x 1}=E_{x 3}=3000 \mathrm{~V} / \mathrm{m}, d \bar{p} / d \bar{z}=0\right.$, $\left.\beta_{1}=\beta_{3}=1\right)$.

Fig.7 Comparison of the velocity profile between transient model when $\bar{t}=10^{-2}$ and the two-fluid experimental data [32] $\left(E_{x 1}=E_{x 3}=-40 \mathrm{~V} / \mathrm{cm}, \beta_{1}=1, \beta_{2}=1.5, \beta_{3}=10^{4}\right)$

Fig.8 Time evolution of $\bar{u}^{\mathrm{p}}, \bar{u}^{E}$ and $\bar{u}$. (a) $\bar{t}=10^{-6}$; (b) $\bar{t}=10^{-5}$; (c)steady state $(d \bar{p} / d \bar{z}$ $=50,000, E_{x 1}=E_{x 3}=3000 \mathrm{~V} / \mathrm{m}, \beta_{1}=\beta_{2}=\beta_{3}=1, \alpha_{1}=\alpha_{2}=\alpha_{3}=1, \bar{\xi}_{3}=\bar{\xi}_{6}=\bar{\xi}_{\text {wall }}$ ).

Fig.9 Time evolution of $\bar{u}^{\mathrm{p}}, \bar{u}^{E}$ and $\bar{u}$. (a) $\bar{t}=10^{-6}$; (b) $\bar{t}=10^{-5}$; (c)steady state $(d \bar{p} / d \bar{z}$ $=50,000, E_{x 1}=E_{x 3}=3000 \mathrm{~V} / \mathrm{m}, \beta_{1}=\beta_{2}=\beta_{3}=1, \alpha_{1}=\alpha_{2}=\alpha_{3}=1, \bar{\xi}_{3}=\bar{\xi}_{6}=0$ ).

Fig.10 Time-dependent velocity, $\bar{u}^{E}$ and $\bar{u}^{\mathrm{p}}$ (a) Within the EDL region (point A of Fig. 3); (b) outside the EDL region of conducting fluid 1 (point B of Fig. 3); (c) within the region of non-conducting fluid 2 (point $\mathrm{C}$ of Fig. 3).

Fig.11 Dimensionless volumetric flow rates for different parameters $\left(E_{x 1}=E_{x 3}=3000\right.$ $\mathrm{V} / \mathrm{m}, d \bar{p} / d \bar{z}=0, K=130$ ).

Fig.12 Dimensionless volumetric flow rates under the effect of electroosmosis and pressure gradient $\left(\beta_{1}=\beta_{2}=\beta_{3}=1, \alpha_{1}=\alpha_{2}=\alpha_{3}=1, \bar{\xi}_{3}=\bar{\xi}_{6}=\bar{\xi}_{\text {wall }}\right)$. 


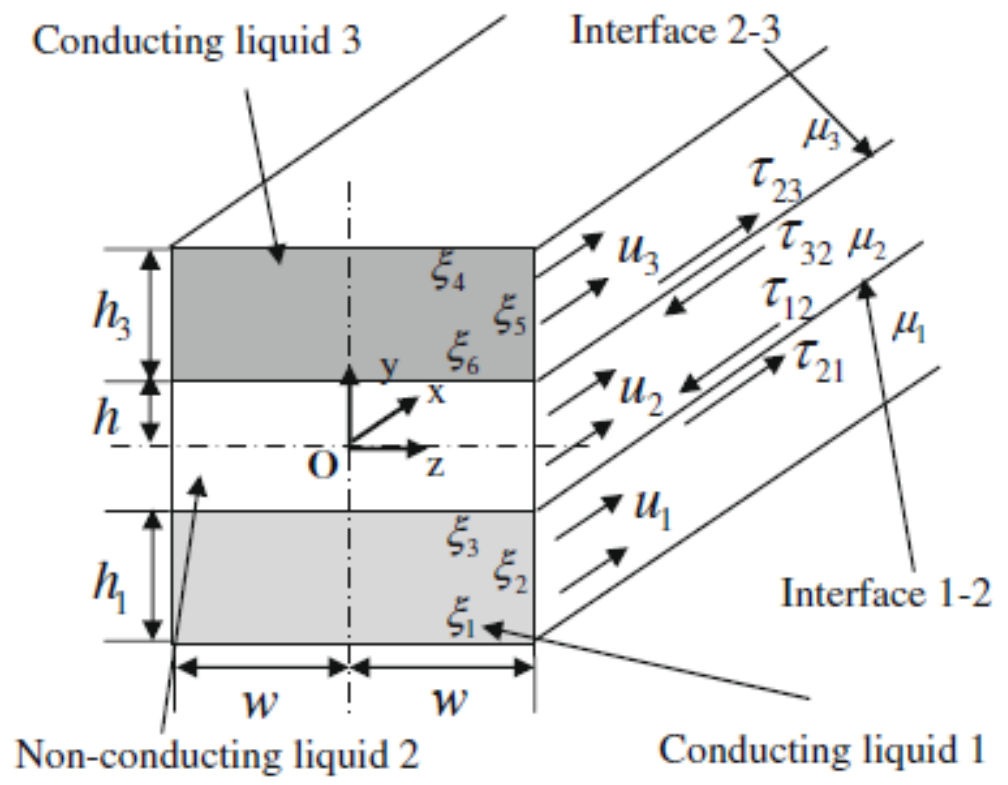

Fig.1 


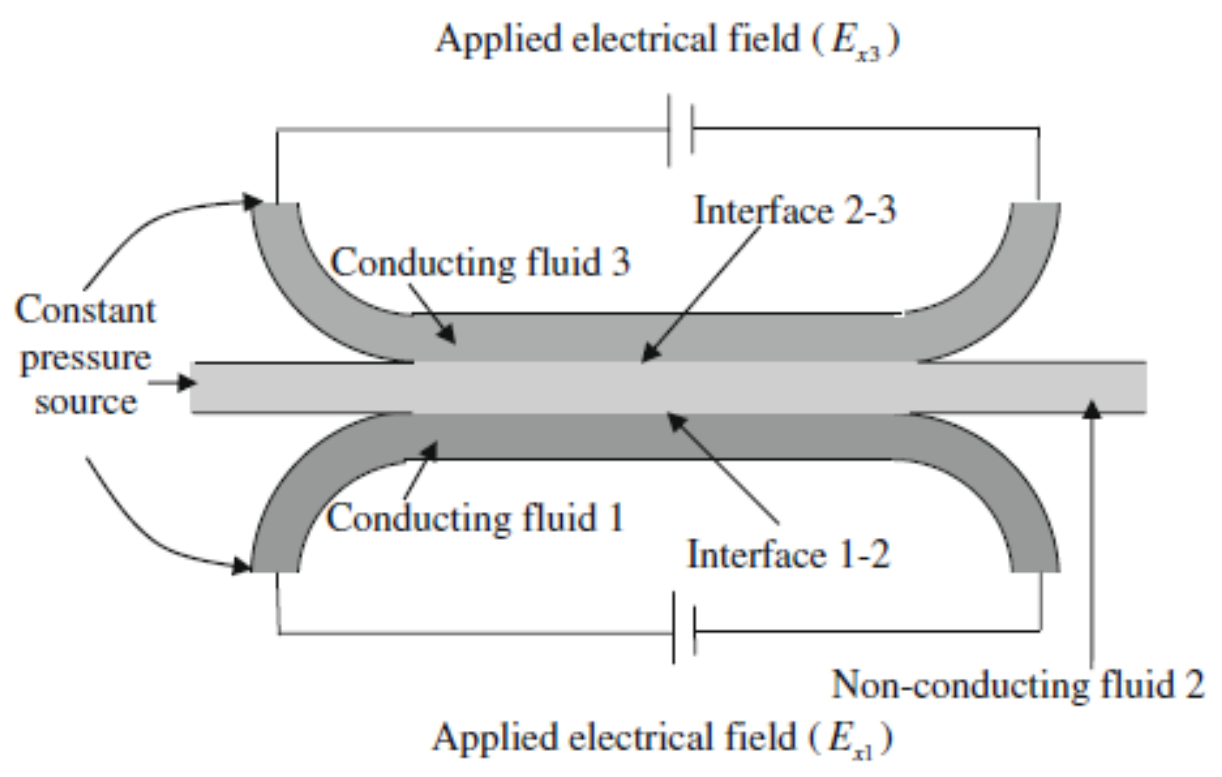

Fig.2 


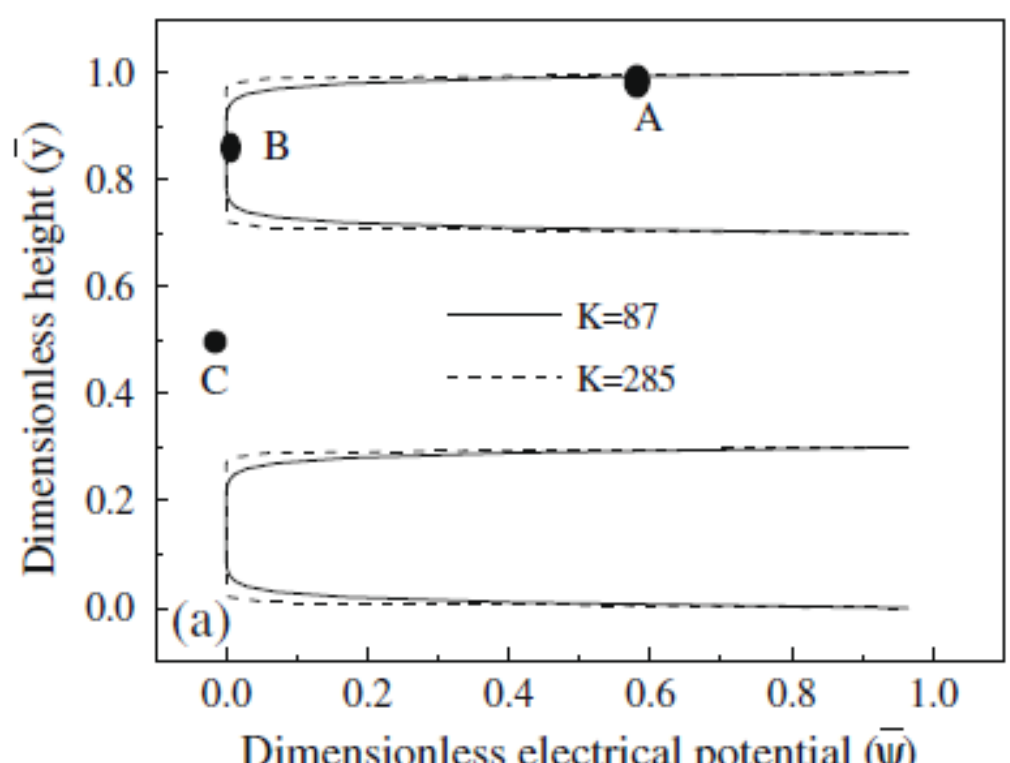

Fig.3 
(a)

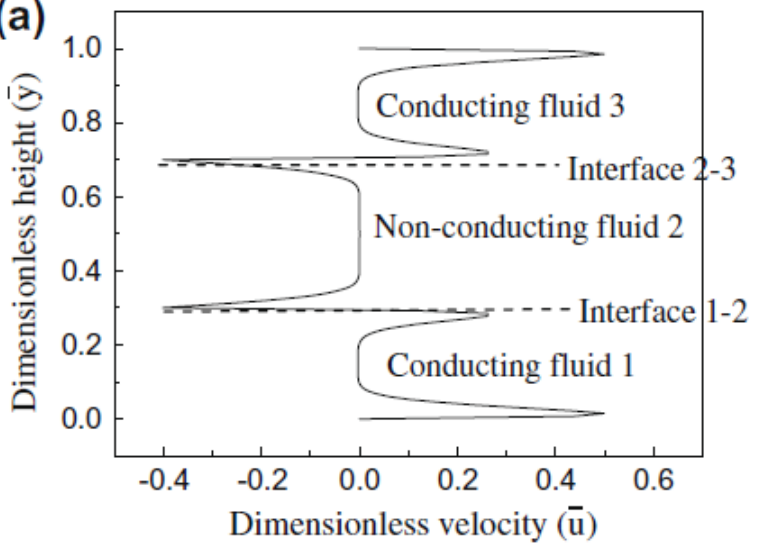

$\bar{t}=1 \times 10^{-6}$

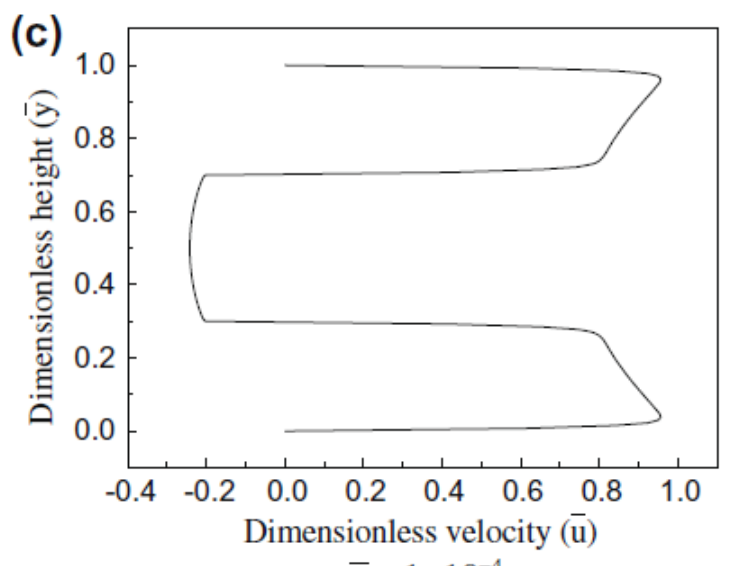

$\bar{t}=1 \times 10^{-4}$ (b)

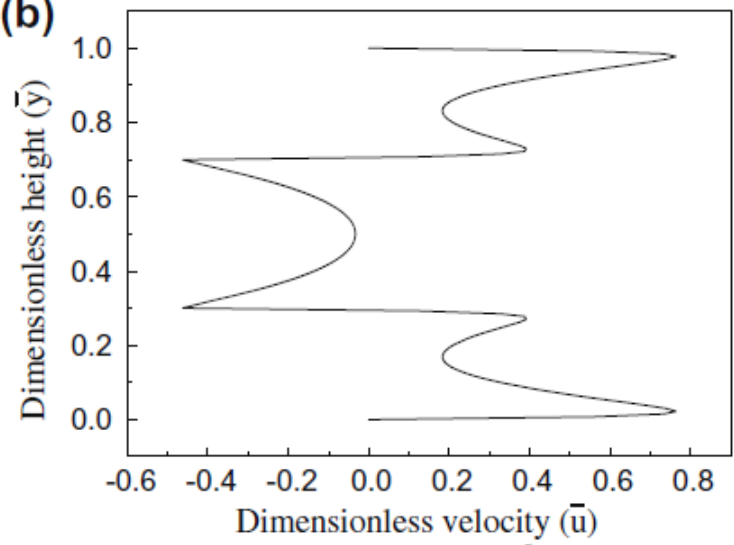

$\bar{t}=1 \times 10^{-5}$

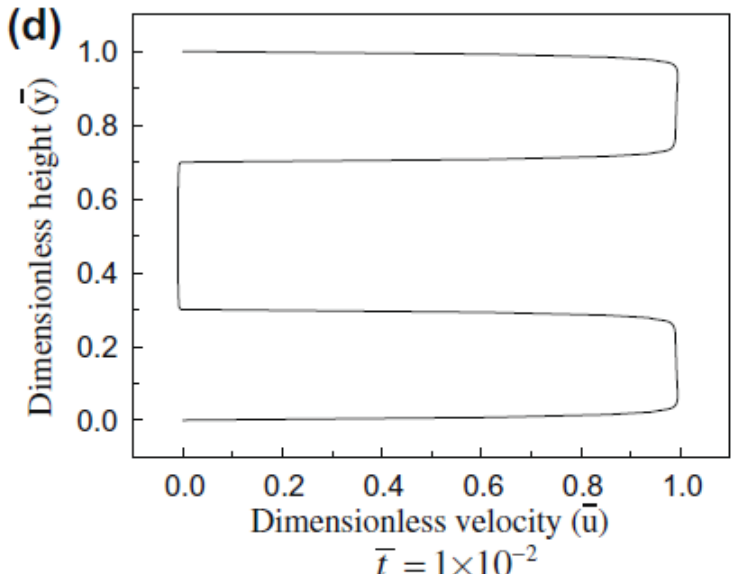

Fig.4 

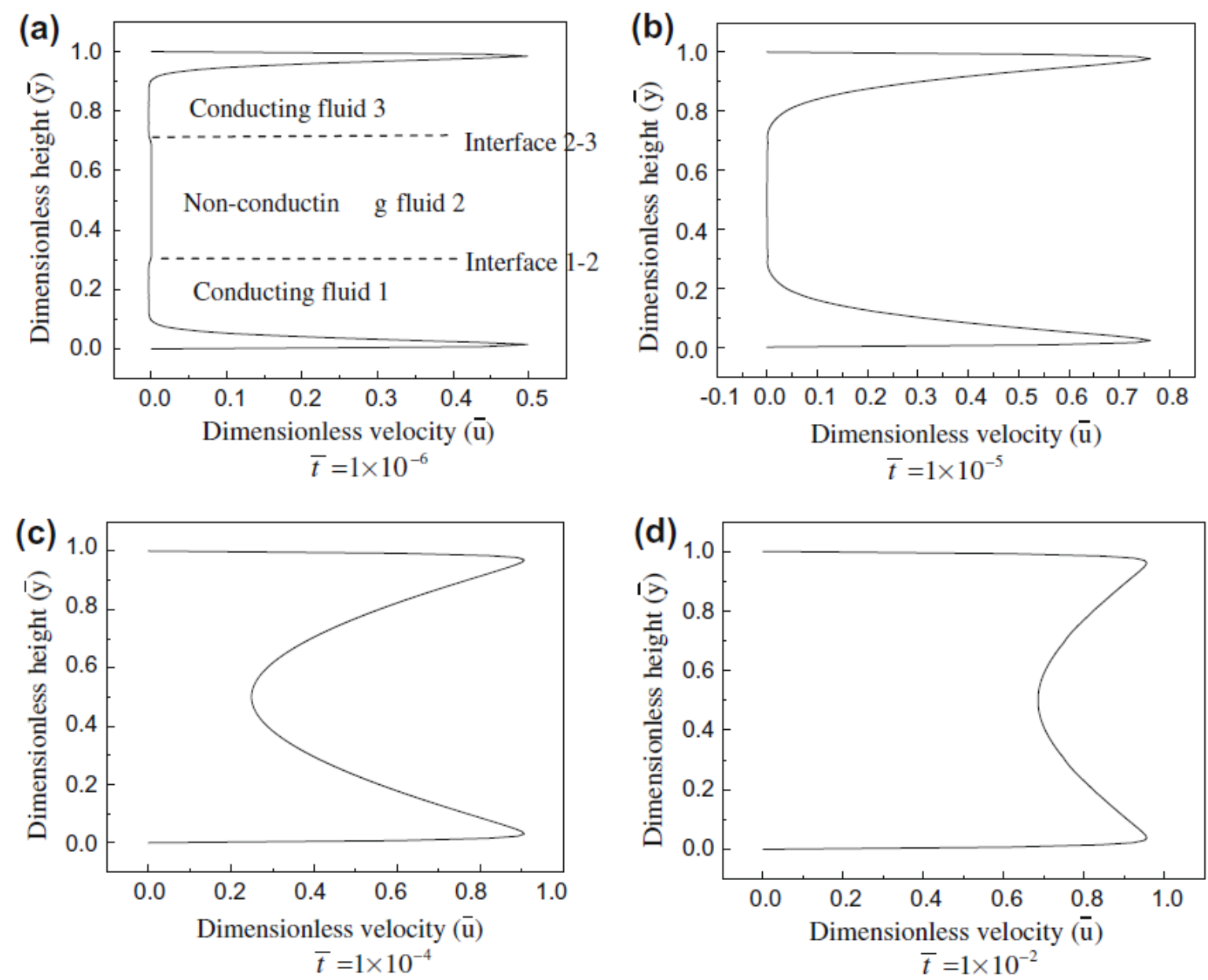

Fig.5 


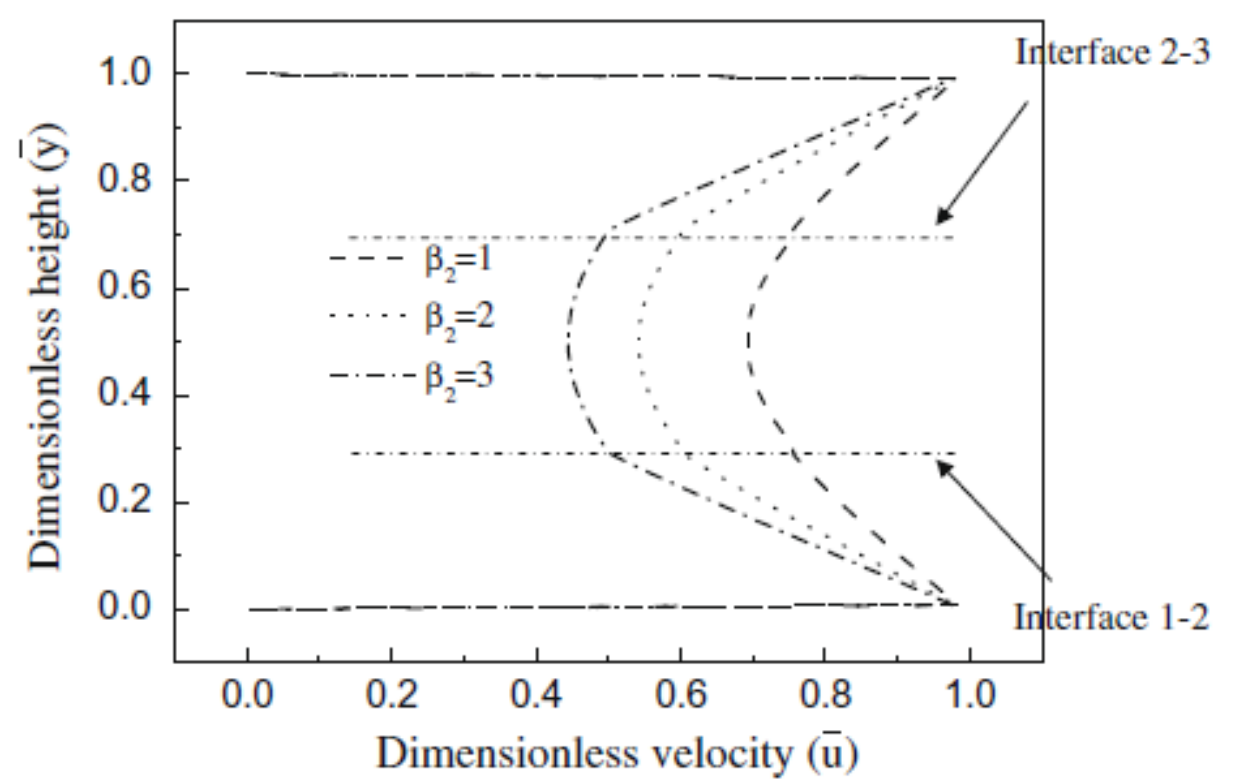

Fig.6 


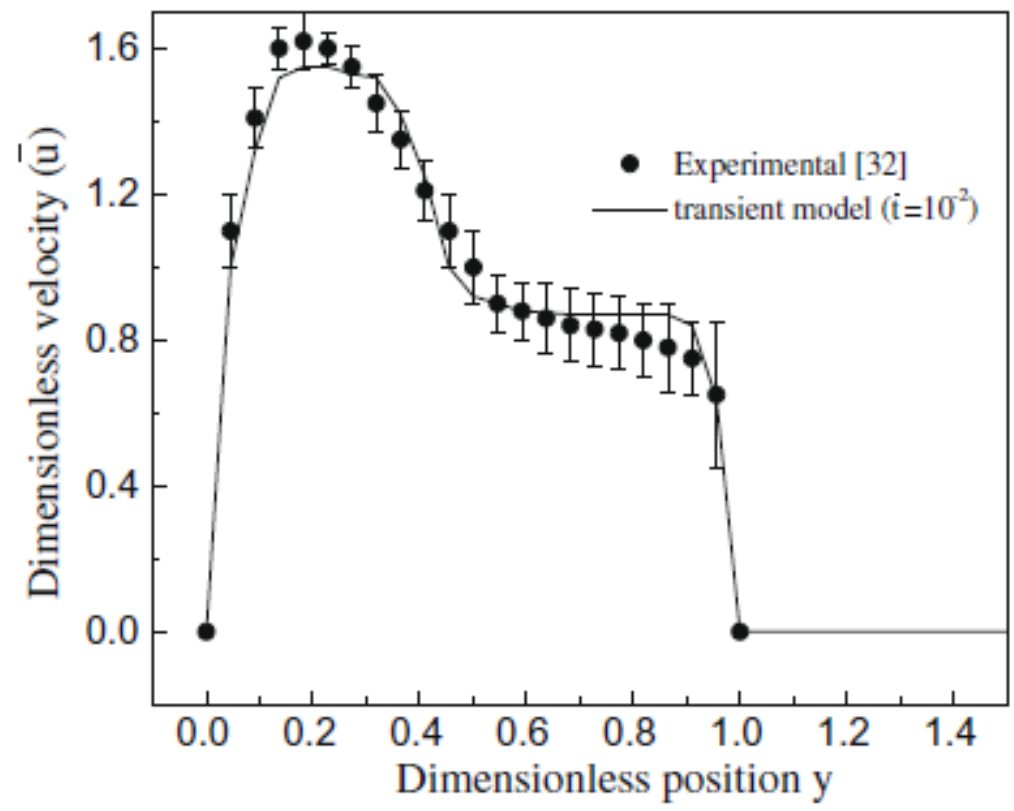

Fig.7 

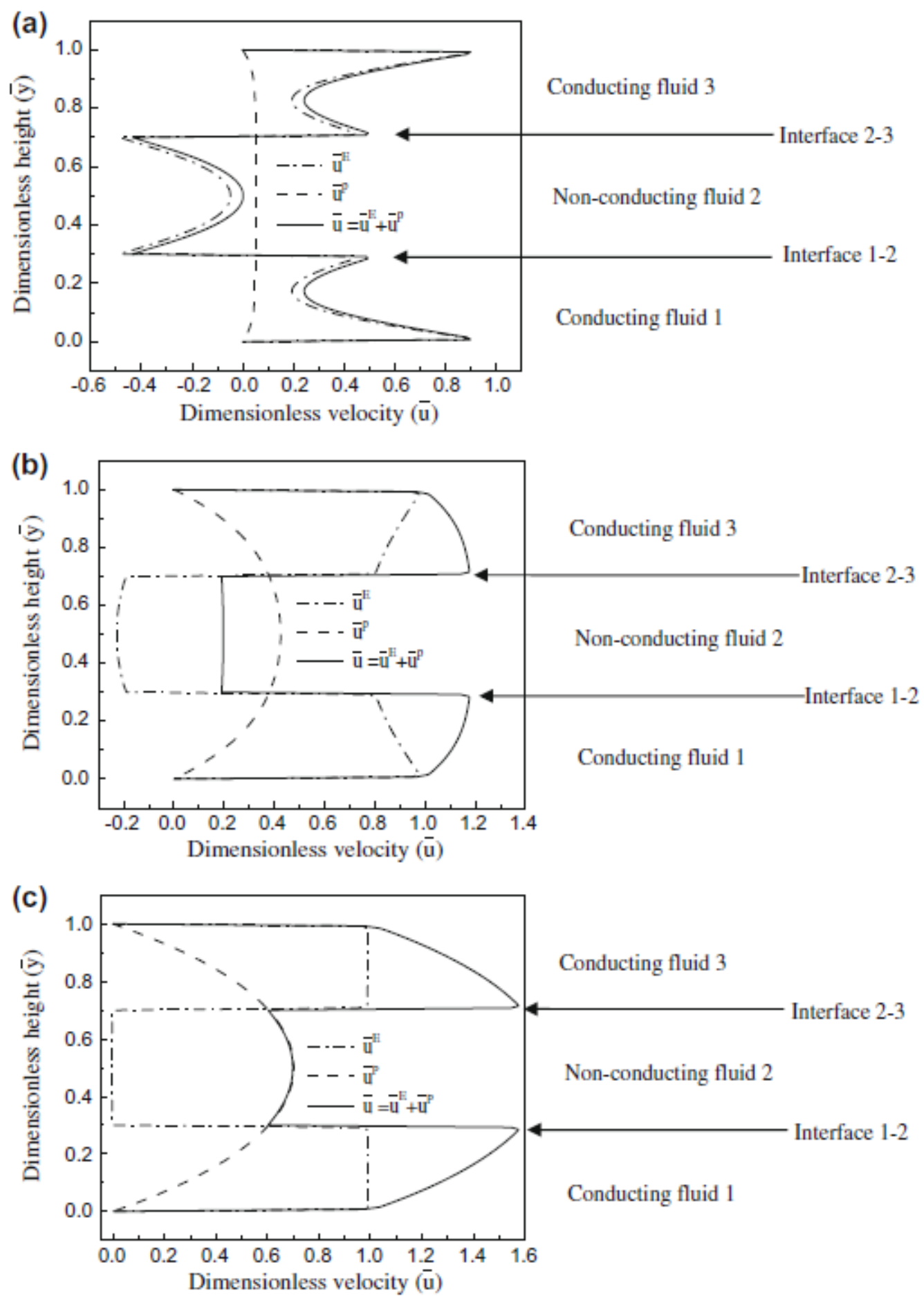

Fig.8 

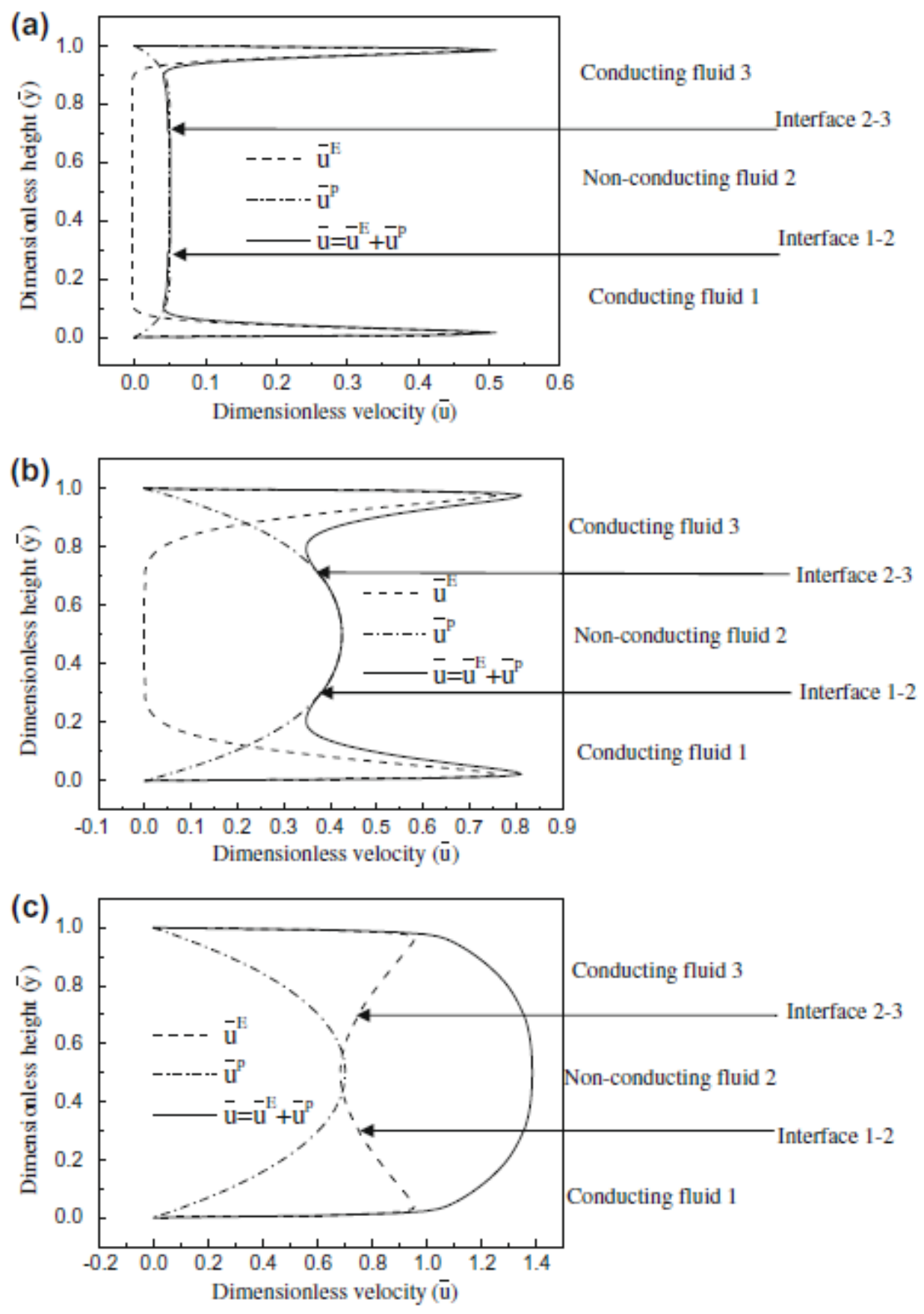

Fig.9 

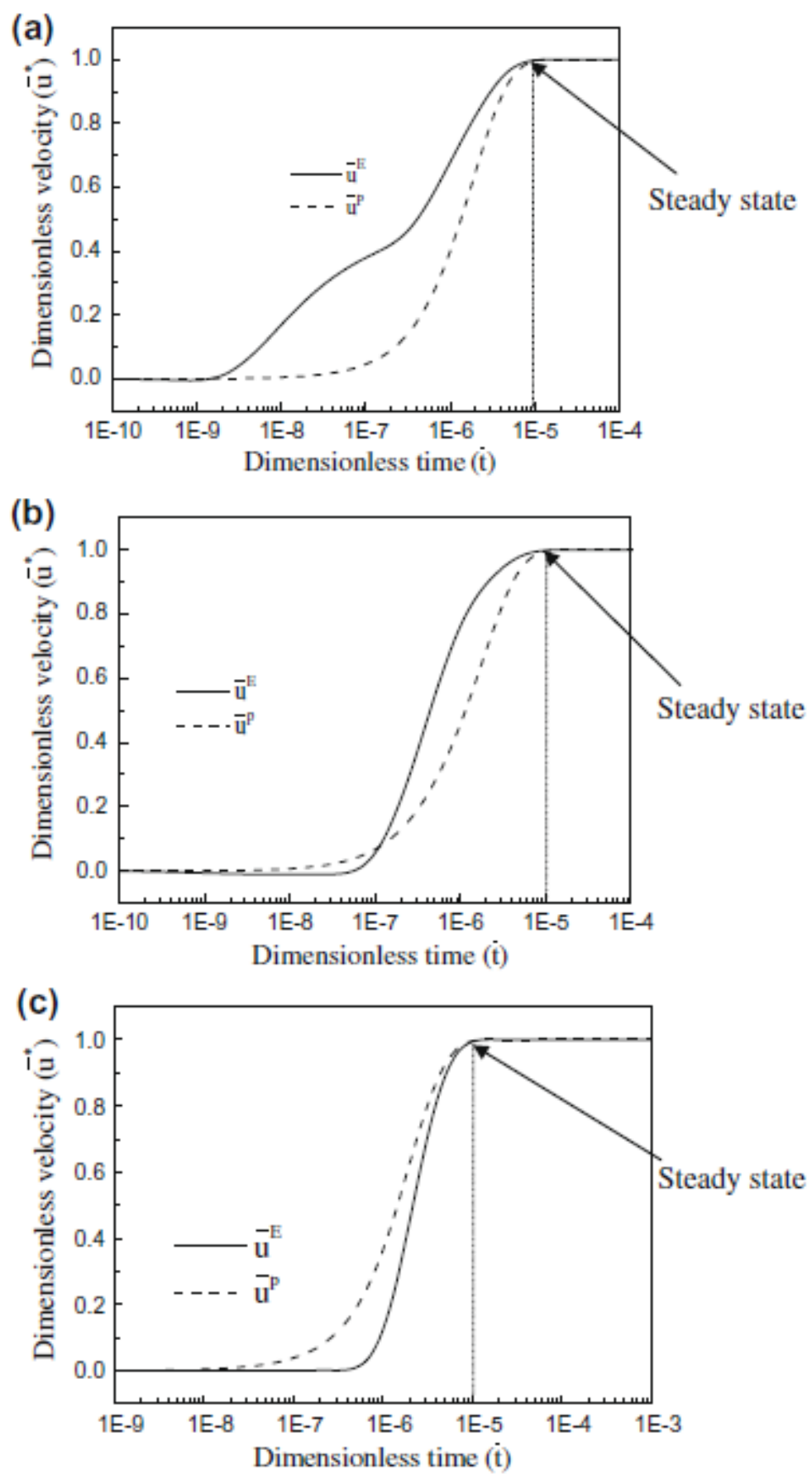

Fig.10 

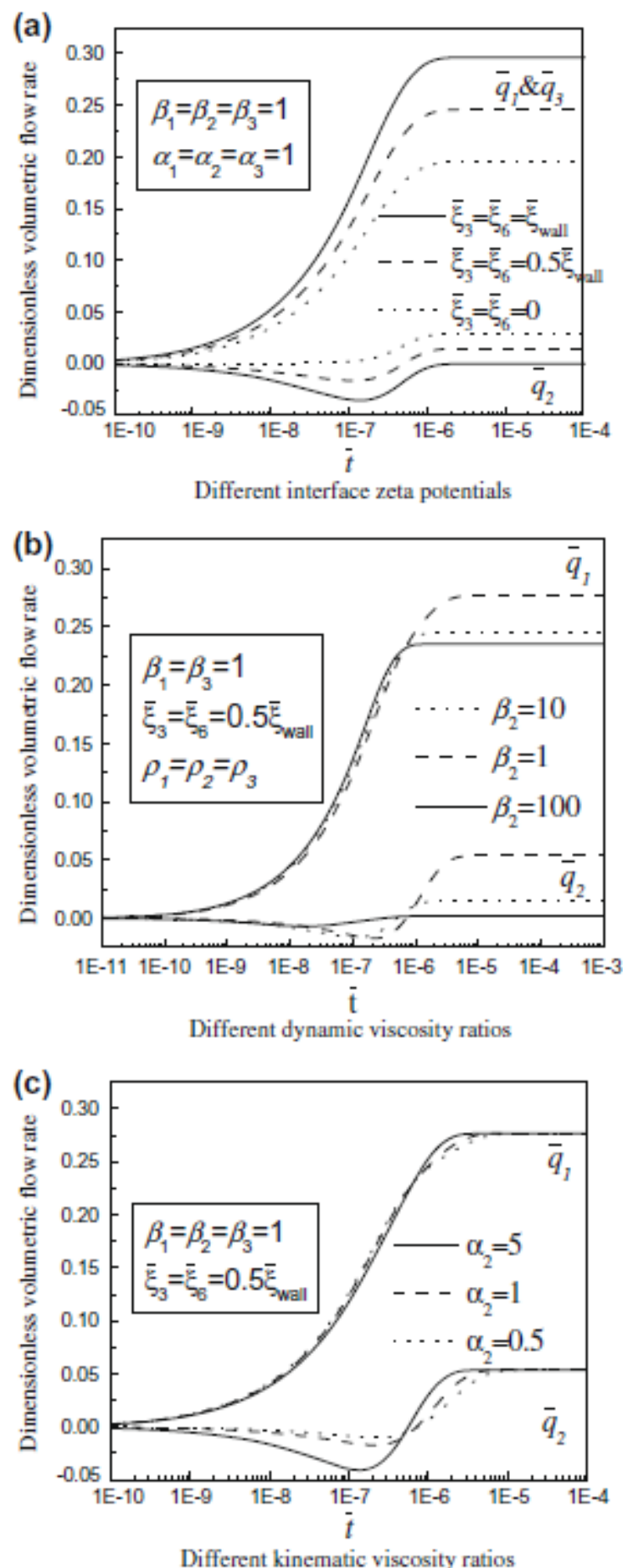

Fig.11 


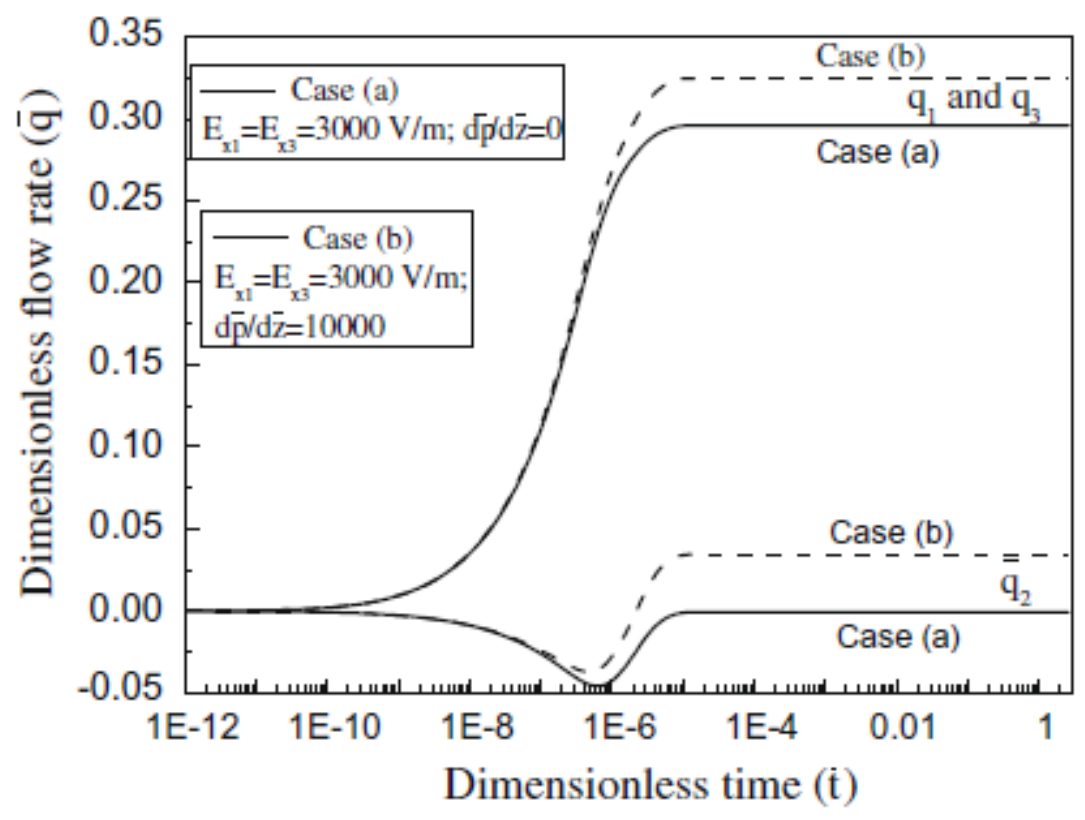

Fig.12 